
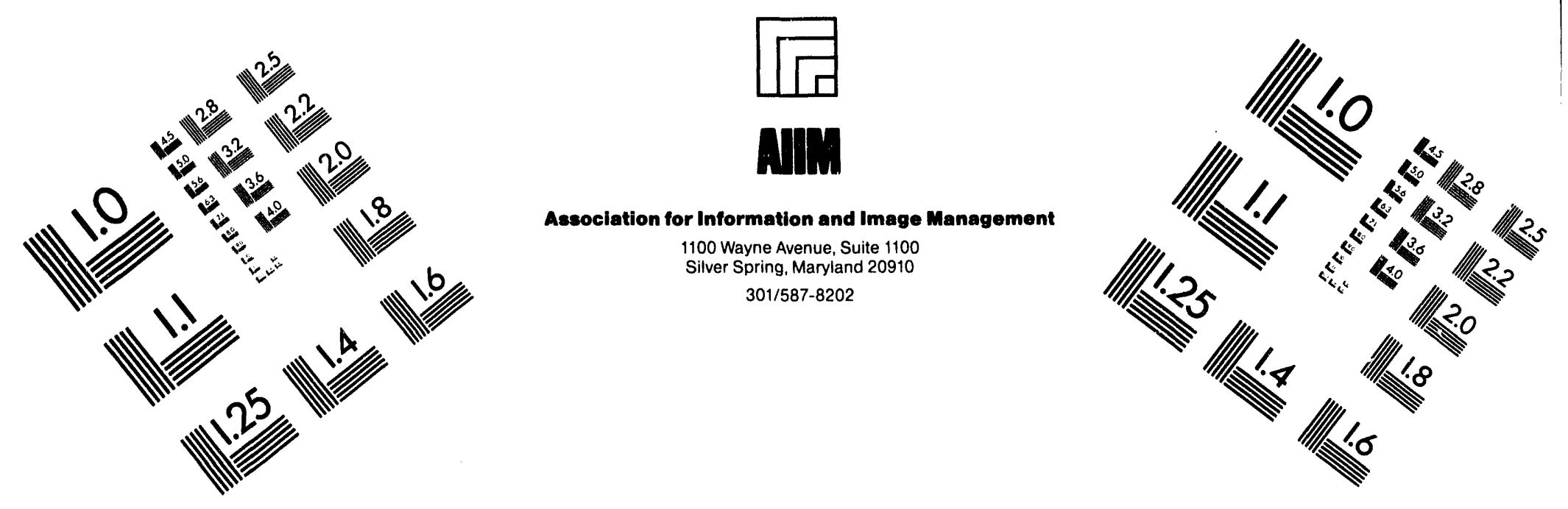

Centimeter

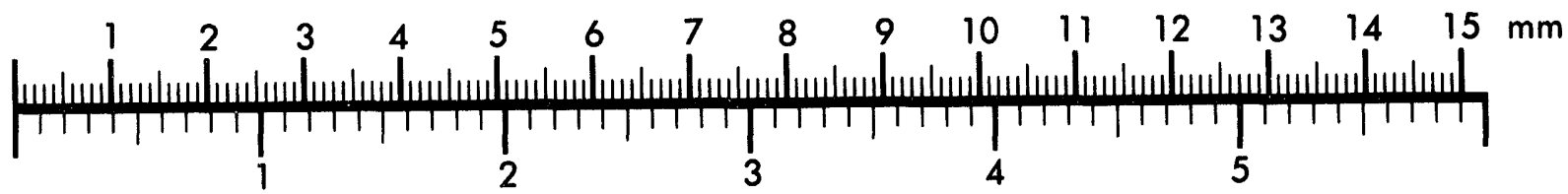
Inches
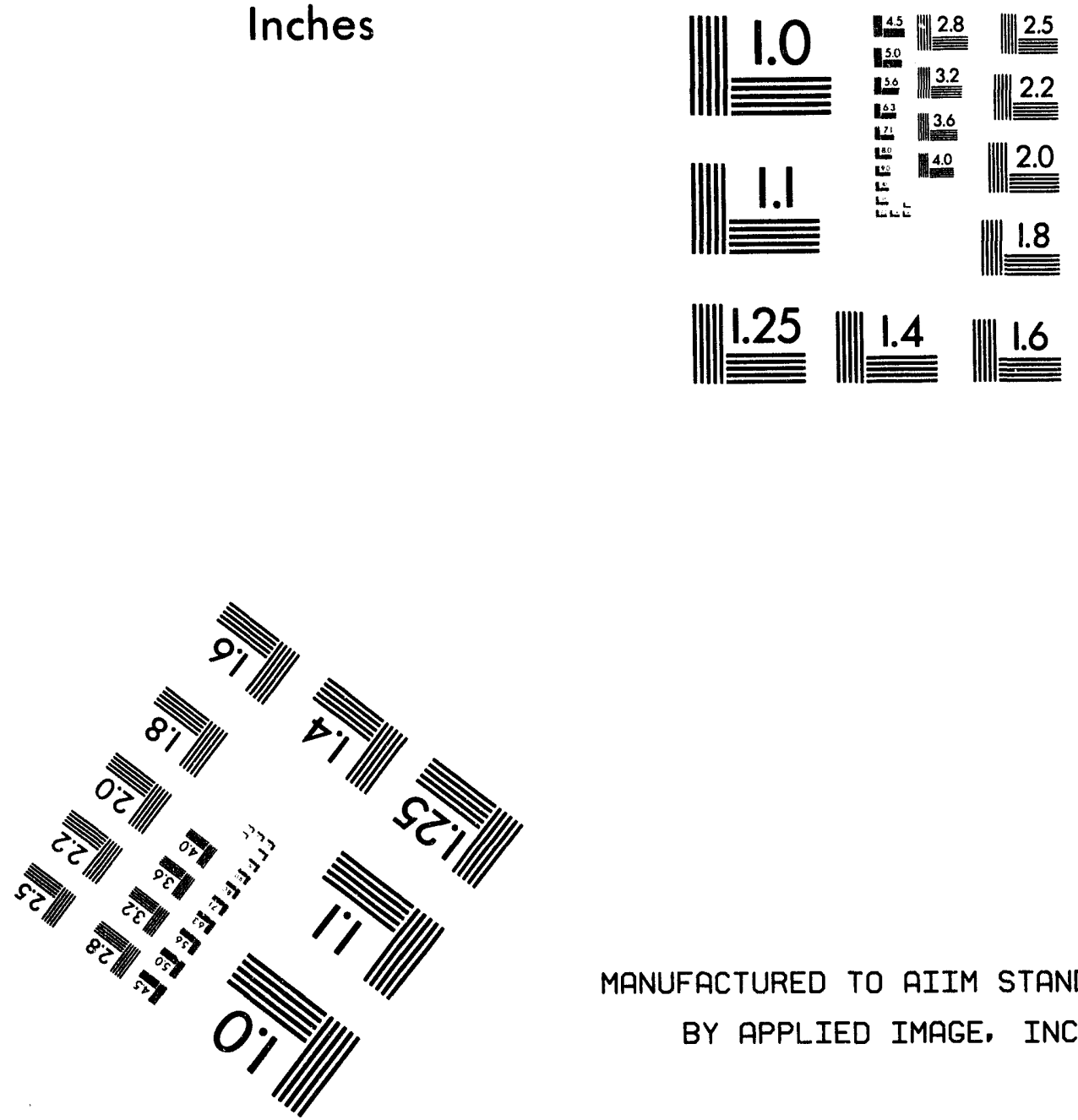

MANUFACTURED TO AIIM STANDARDS

BY APPLIED IMAGE, INC.

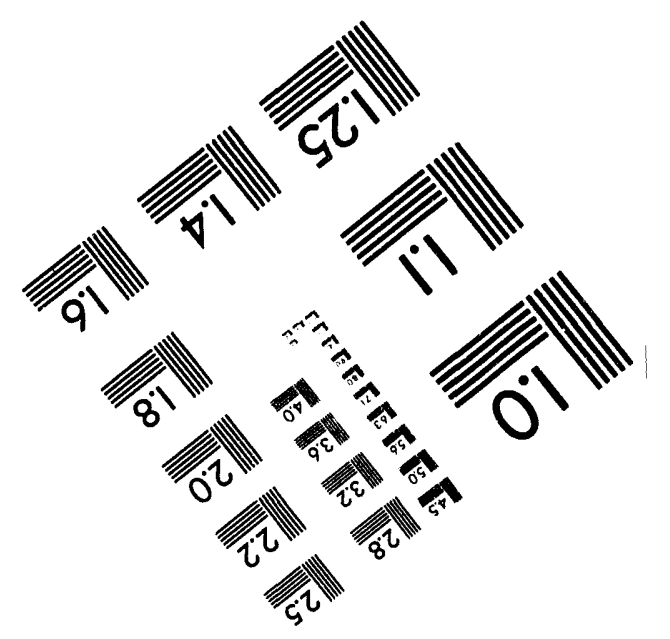



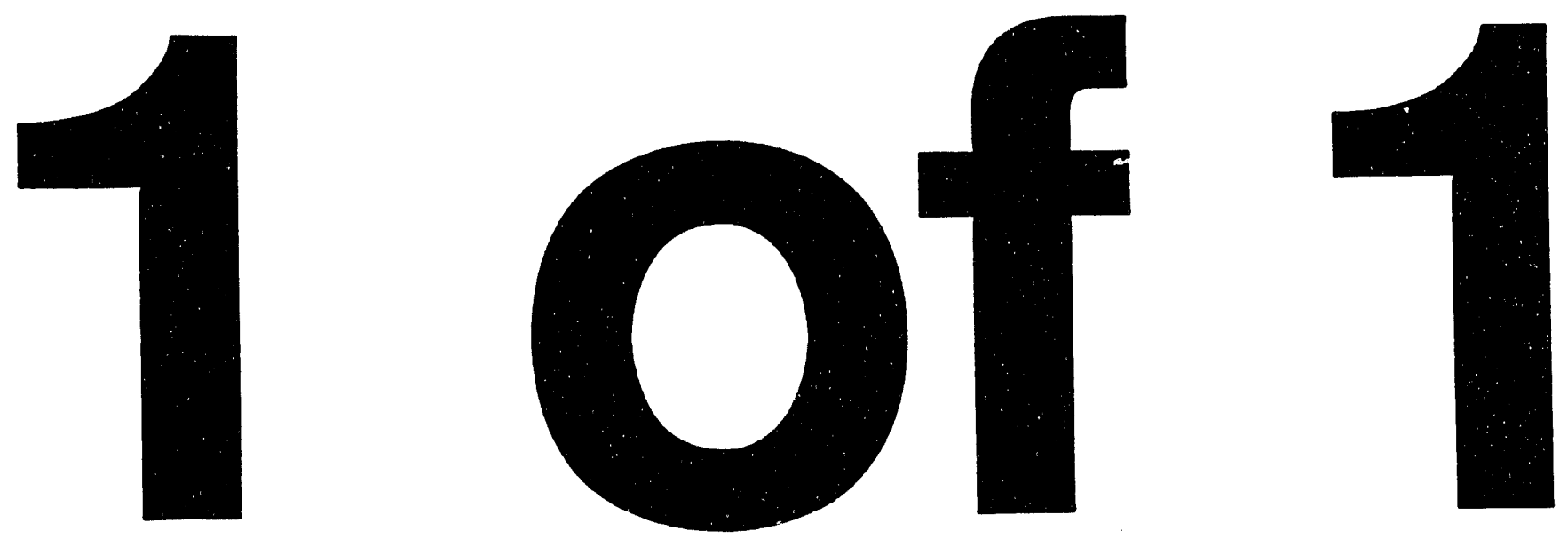


\section{To: (Receiving Organization) \\ Distribution}

5. Proj./Prog./Dept./Div.: Hydrogen Mitigation

8. Originator Remarks:

Transmittal of Design Review Report for Multiport Riser

Final Design Review for approval and distribution. Engineering Support
4. Related EDT No.:

$N / A$
6. Cog. Engr.
D. R. Precechtel
7. Purchase Order No.:
$\mathrm{N} / \mathrm{A}$

9. Equip./Component No.: $N / A$

10. System/Bldg./Facility: 241-SY-101

11. Receiver Remarks:
12. Major Assm. Dwg. No.: $N / A$

13. Permit/Permit Application No.: $N / A$

14. Required Response Date:

$N / A$

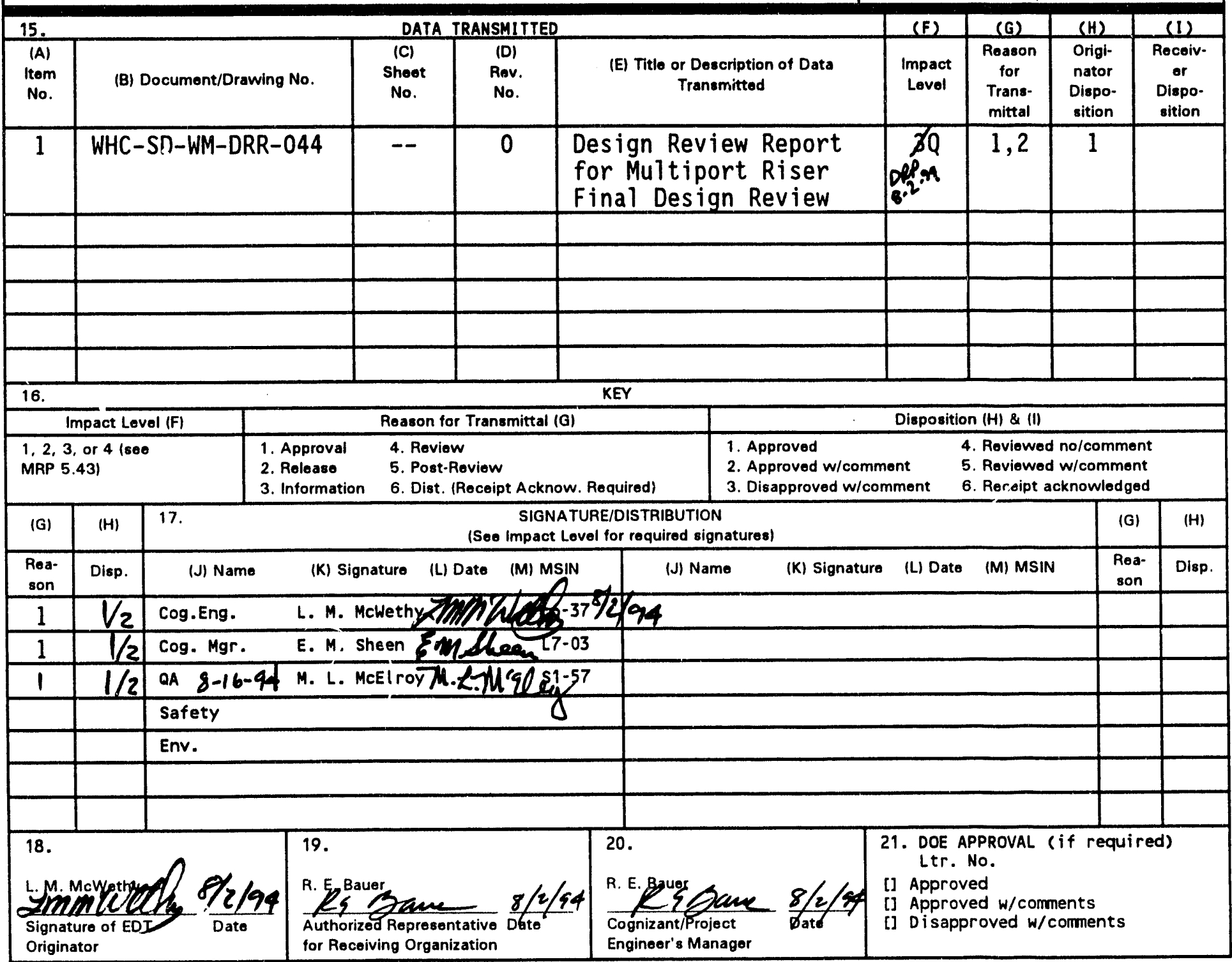




\section{RELEASE AUTHORIZATION}

\section{Document Number: WHC-SD-WM-DRR-044, REVISION 0}

Document Title: Design Review Report for Multiport Riser Final Design Review

Release Date: $\quad 8 / 18 / 94$

This document was reviewed following the procedures described in WHC-CM-3-4 and is:

APPROVED FOR PUBLIC RELEASE

***************

WHC Information Release Administration Specialist:

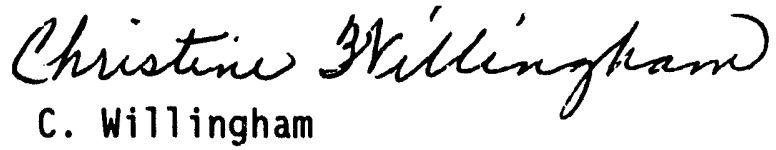

$8 / 23 / 94$

(Signature)

(Date) 
2. Title

Design Review Report for Mulijport Riser Final Design Review

\section{Key Words}

Multiport

SY-101

Mitigation

Tank

Waste
3. Number

WHC-SD-WM-DRR-044 4. Rev No.

7. Abstract

6. Author

Name: L. M. McWethy

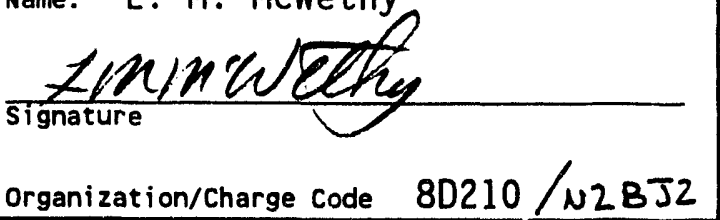

Reports the conclusion of the final design review for Multiport Riser.

\section{APPROVED FOR \\ OURPOSE AND USE OF DOCUMENT - This} be U.S. Department of Energy and its contractors.

y to perform, direct, or integrate under U.S. Departlue of Energy contracts. This document hot approved for public releastonitil reviewed.

PATENT STATUS - This docuin compince it is transmitted in advance of patent clearance, in confidence solely for use in performane of work mader contracts with the U.S. Department of En-ryy. This document pungt to be published nor its contents otbuthise disseminated or used folmurposes other than specified ste before patent approval for such reture or use has been soyfted, upon request, from the Patent Counsel, U.S.martment of rgy Field office, Richland, WA.

DISCLAIMER - This report was prepared as an account of work sponsored by an agency of the United States Government. Neither the United States Government nor any agency thereof, nor any of their employees, nor any of their contractors, subcontractors or their employees, makes any warranty, express or implied, or assumes any legal liability or responsibility for the accuracy, completeness, or any third party's use or the results of such use of any information, apparatus, product, or process disclosed, or represents that its use would not infringe privately owned rights. Reference herein to any specific commercial product, process, or service by trade name, trademark, manufacturer, or otherwise, does not necessarily constitute or imply its endorsement, recommendation, or favoring by the United States Government or any agency thereof or its contractors or subcontractors. The views and opinions of authors expressed herein do not necessarily state or reflect those of the United States Government or any agency thereof.

9. Impact Level $\$ Q$

$$
\text { DRP } 8 / 2 / 94
$$




\title{
DESIGN REVIEW REPORT \\ FOR MULTIPORT RISER FINAL DESIGN REVIEW
}

\author{
L. M. McWethy \\ Design Review Secretary \\ E. M. Sheen \\ Design Review Chairman
}

May 1994

\section{DISCLAIMER}

\begin{abstract}
This report was prepared as an account of work sponsored by an agency of the United States Government. Neither the United States Government nor any agency thereof, nor any of their employees, makes any warranty, express or inplied, or assumes any legal liability or responsibility for the accuracy, completeness, or usefulness of any information, apparatus, product, or process disclosed, or represents that its use would not infringe privately owned rights. Reference herein to any specific commercial product, process, or service by trade name, trademark, manufacturer, or otherwise does not necessarily constitute or imply its endorsement, recommendation, or favoring by the United States Government or any agency thereof. The views and opinions of authors expressed herein do not necessarily state or reflect those of the United States Government or any agency thereof.
\end{abstract}




\section{CONTENTS}

1.0 scope ........................ 3

2.0 Summary ...................... 3

3.0 Documentation ................... 4

\section{Attachments}

1 Letter, J. R. Frederickson to Design Review

Committee, "Multiport Riser Final Design Review," dated February 28, 1994 . . . . . . . . . . . . . . . . 6

2 Review Comment Record Database for Final

Design Review.................. . 10

3 Memo, L. M. McWethy to MPR Design Review

Committee, "Approval for Fabrication of

Multiport Riser Assembly," dated May 11, 1994 . . . . . . . . . . . 40

4 Memo, L. M. McWethy to MPR Design Review

Committee, "Completion of Multiport Final

Design Review," dated June 14, 1994 . . . . . . . . . . . . . . . 52

5 Design Review Committee's Approval

6 Design Review Checklist . . . . . . . . . . . . . . 57

7 Agenda and Member Assignments for Final

Design Review..... . . . . . . . . . . . . 64

$8 \quad$ Plans for the Final Design Review . . . . . . . . . . . . 67 


\section{DESIGN REVIEW REPORT \\ FOR MULTIPORT RISER FINAL DESIGN REVIEW}

\subsection{SCOPE}

This design review examined the design documents contained in a notebook prepared for the final design review of the multiport riser assembly. The notebook was titled: "Multiport Riser Final Design Review."

This notebook was dated March 16, 1994 to coincide with the convening date of the formal design review meeting. This document was distributed March 1, 1994 to the design review committee and others by J. R. Frederickson via formal correspondence (Attachment 1). This letter stated the purposes and intentions of the review.

\subsection{SUMMARY}

The review was conducted using a database comment registration system whereby the reviewer comments were entered into the database and each comment was given a unique identifier. During the review, each comment was referred to by its identifier. Changes to comments, responses, and actions needed were made with the agreement of the committee and the reviewers at the meeting. Some comments were closed at the meeting with the concurrence of the committee. The remaining comments required specific analyses and documentation to be completed. The persons responsible for resolving these comments were identified. The complete review comment record (RCR) database is enclosed as Attachment 2. This record will form one of the bases for the final acceptance of the multiport riser assembly at the operational readiness review. This record will assure design continuity and maintain the history of all comments, responses, and resolutions.

At the final design review, the structural analyses were not available. The design review committee needed these analyses to complete its review and approve the multiport assembly for fabrication. It was agreed that approval for fabrication would require completion of the design review checklist and review of a summary of the structural analysis results. This was requested of the committee in a May 11, 1994 memo (Attachment 3). The summary results of the structural analyses were not adequate to complete the design review. When the structural analyses were available, they were distributed for review (Attachment 4). This allowed closure of all design review comments. Approval of the design was achieved and is shown in Attachment 5. The completed design review checklist is shown in Attachment 6 . The design review committee members are shown in Attachment 7, and the plans for the design review are shown in Attachment 8. 

committee.

This approval completes the responsibilities of the design review

\subsection{DOCUMENTATION}

Documents attached to this report describing the status, approvals, and history of the final design review are:

\section{Attachment 1}

Letter, J. R. Frederickson to Design Review Committee, "Multiport Riser Final Design Review," 9451465, dated February 28, 1994.

Officially transmitted the design review notebook to the design review committee and other interested parties.

\section{Attachment 2}

Review Committee Record (RCR) Database for the Multiport Riser Final Design Review.

This record contains six comments remaining from the 60 percent design review. of the 63 comments received at the final design review, 36 are completed. The remaining comments were completed after review of the structural analyses report.

\section{Attachment 3}

Memo, i. M. McWethy to MPR Design Review Committee, "Approval for Fabrication of Multiport Riser Assembly," dated May 11, 1994.

This transmitted the summary results of the structural analyses and the design review checklist. Review and approval for fabrication was requested.

\section{Attachment 4}

Memo, L. M. McWethy to MPR Design Review Committee, "Completion of Multiport Final Design Review," dated June 14, 1994.

This transmitted the results of the structural analyses. Review and approval of the MPR design was requested.

\section{Attachment 5}

Signature Approval of the Design Review Committee, Approving the Multiport Design for Fabrication. 


\section{Attachment 6}

Design Review Checklist, as Approved by Design Review Committee.

This approved checklist was signed by the Chairman, Secretary, and Quality Assurance representative of the committee.

\section{Attachment 7}

Agenda and Committee Member Listing for the Final Design Review.

\section{Attachment 8}

Plans for the Design Review - Distributed at the Kick-off Meeting of March 1, 1994.

This included the agenda, design review committee assignments, and the instructions for conducting the review and documenting comments. 
WHC-SD - WM-DRR- 044

Rev. 0

Page 6

ATTACHMENT 1 


\section{CORRESPONDENCE DISTRIBUTION COVERSHEET}
Author
Addressee
Correspondence No.
J. R. Frederickson
Design Review Committee
9451465

Subject: MULTIPORT RISER FINAL DESIGN REVIEW

INTERNAL DISTRIBUTION

Name

Location

w/att

Name

Location

$w / a t t$

Correspondence Control

A3-01

R. E. Mendoza

$R 1-51 * \quad X$

H. E. Adkins

N1-45* X

D. R. Precechtel

L6-37

$x$

T. R. Benegas

H5-09

R. W. Reed

RI-51

J. R. Biggs

T4-07* X

C. C. Scaief

L7-06* $x$

N. S. Cannon

L6-38

E. W. Sheen

L7-03* $X$

J. B. Crystal

Ni-42 $X$

T. I. Stokes

H5-09 X

L. E. Efferding

HO-33* X

C. B. Thomas

$\mathrm{N} 1-03$

$x$

J. R. Frederickson (2)

N1-42 X

R. W. Truitt

N1-42

G. J. Gauck

R1-51

G. F. Vargo

H5-09 X

L. H. Goldmann

$\mathrm{N} 1-42$

SPMD File

$\mathrm{N} 1-42 \quad \mathrm{X}$

J. I. Gould

$H 6-30 * \quad X$

B. W. Hall

G6-16

M. N. Hall

N1-42 X

C. E. Hanson

H5-09 X

J. J. K1os

T4-07* $X$

L. S. Krogsrud

R3-08* X

R. E. Larson

$S 2-24 * \quad X$

J. W. Lentsch

R2-78 $X$

R. S. Marlow

H5-56* X

M. L. McElroy

$51-57^{*} \quad X$

L. M. McWethy

$\left\llcorner 6-37^{*} \quad X\right.$

* Design Review Committee Members 


\section{OFFSITE}

Los Alamos National Laboratory University of California Post Office Box 1663

Los Alamos, New Mexico 87545

R. P. Johnson (4) H5-09

\section{ONSITE}

Pacific Northwest Laboratory

J. R. Friley

K5-22*

N. R. Gordon

$\mathrm{K} 2-44 *$

w/o Attachment

C. W. Stewart

$K 7-15$

*Design Review Committee Members 
P.O. Box 1970 Richland, WA 99352

February 28, 1994

9451465

Design Review Committee:

MULTIPORT RISER FINAL DESIGN REVIEW

Attached is the design documentation for the Multiport Riser system. This system will support the Hydrogen Mitigation Test program for mitigation of safety issues for Hanford Waste Tank 241-SY-101. The purpose of the Multiport Riser is to provide multiple access ports of several diameters in a single 42" riser for simultaneous access of test and support equipment. The design is complete, subject to verification by this design review.

Please review and comment on the attached design information for a design review to be held at 8:00 a.m., March 16, 1994 in the 4862 Building (FMEF), Conference Room 1. This review will assure that all design requirements are met and that fabrication of this system can proceed.

Please provide your review comments to Marci Clayton by March 14, 1994 so they can be placed in a database for the review meeting. Comments can be submitted by cc:Mail, sent to her at MSIN N1-42, or faxed to 373-0209.

If you have any questions regarding your review, please contact Ed Sheen, Review Chairman, on 376-5117, Mike McWethy, Review Secretary, on 376-9507, or myself on 376-0691.

Very truly yours,

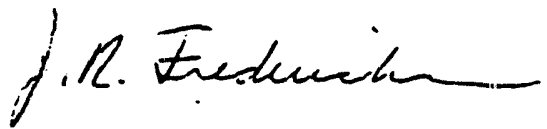

J. R. Frederickson, Manager Process Systems Development Engineering Development

mac

Attachment 
WHC-SD-WM-DRR-044

Rev. 0

Page 10

ATTACHMENT 2 


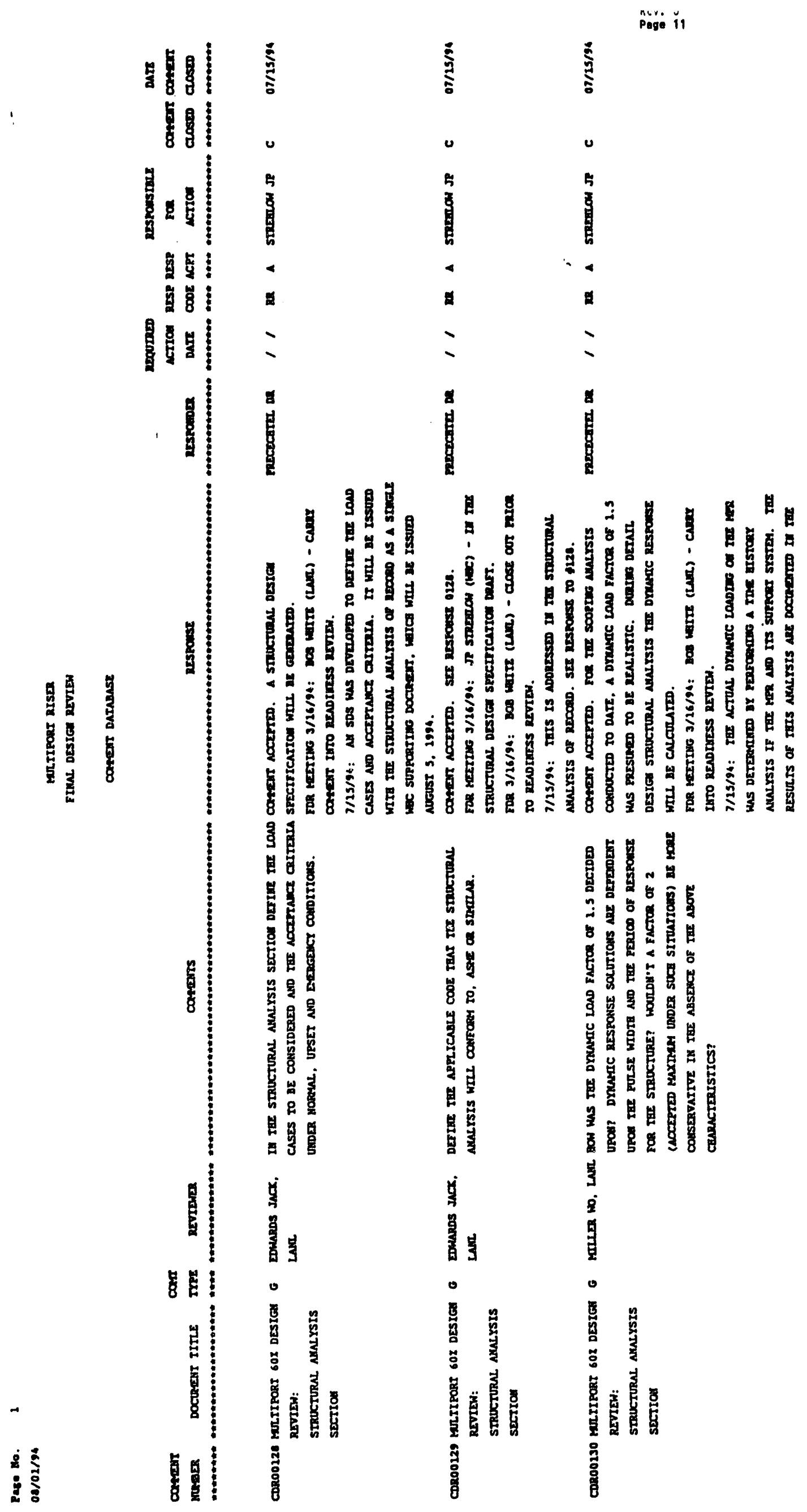


Pase Ho. 2

08/01/94

Merifront RIsER

FIMLL DESIGA REVIEH

Congar: DATAMASE

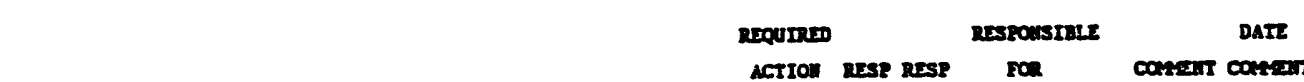

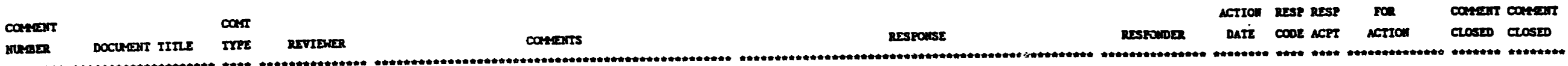

suestex

YYE

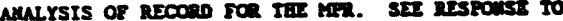

128.

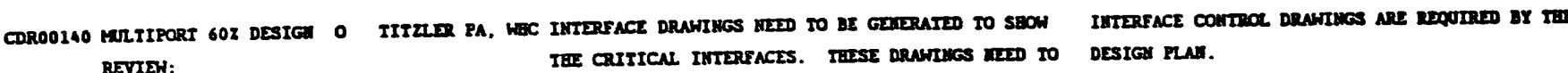

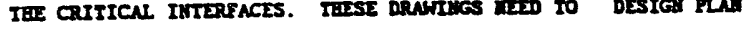

InTERFACE CONTROR

DRAHTHGS

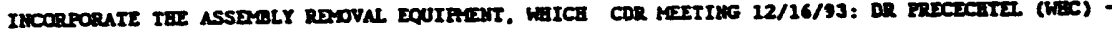

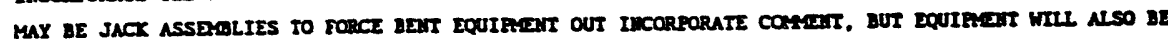

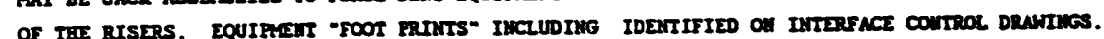

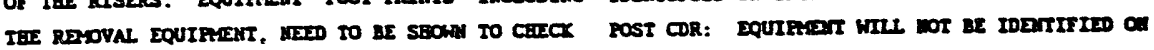

OUT IMTRRs

CDroost3 hertiport 602 Destor

RETIEN:

SIRUCTURNL ALULYSIS

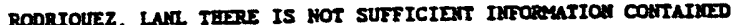

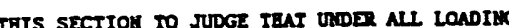
Combitrous THAT THE DESIGA IS ADPQunTE.

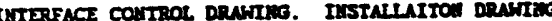

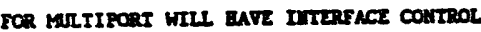

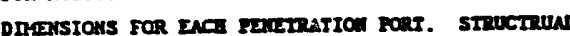

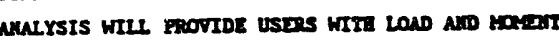

LIMTIS FOR EACB RORT. THIS DATA WTLL BE UTILIZT

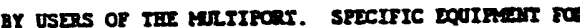

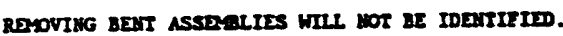

FOR HERTIMG 3/16/96: JR TreDertcrson (waC) -

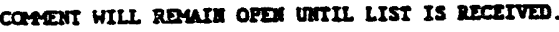

7/25/94: NOTE a ans ADOED TO TII InStallation

DRAWING, E-2-821100, To REQULEE IIII USERS Of TII

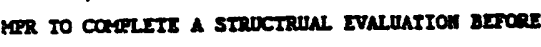

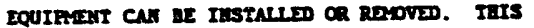

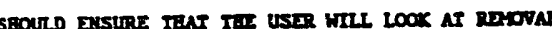

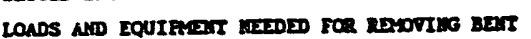

Eournesart.

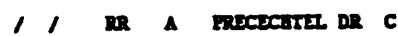

$07 / 15 / 94$

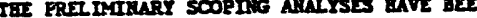

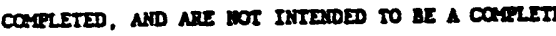
STRUCT⿱R⿴囗丨

CDR MIIIING 12/16/93: IMCORPORATE INTO RESPONSE
Precratrer or , , RR A smerion JP C $07 / 15 / 94$ 
COMgars DATABasE

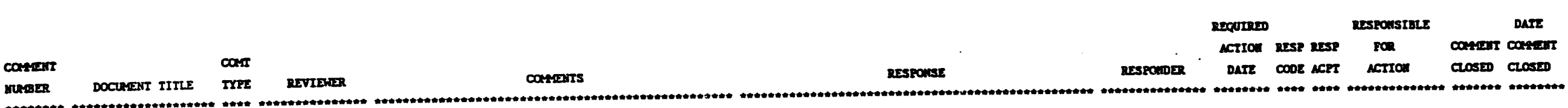

0128.

TOR FEETIMG 3/26/94: DR ERECDCBTII (WAC) -

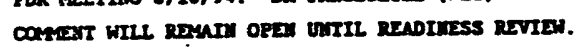

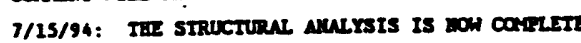

CDRO0174 MarTIPORT 60Z DESIG REviEN:

STRUCTURA ANRLYSIS

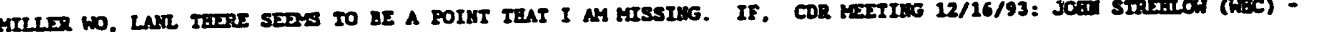
FOR RISER A, 100,000 LBF IS USED TO OPPOSE III GAS MABOINS APPEAR TO BE ADEOUATE, CEARIFICATIOA WTHL LOAD, ND IBIS IMCLUDES IEE WEIGBS OF IEI CONCRETE BE FUT IV ARALYSTS.

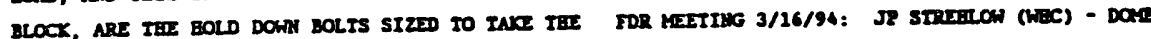

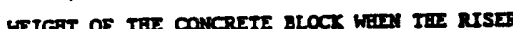

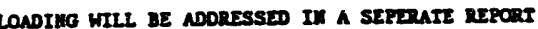
HEIGET OF IBE CONCREIT BLOCX WEIN III RISER ASSDABLY TRITS TO LIFT OEF? THIS APPEARS TO BE TII PFOBLDY CITID FOR RISER 8. II: TIE DOLTS DO nOT BAVE SUFEICIERT STRENGTS. ALSO, THE 100,000

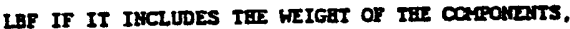
IE: IBI SOAIC PROBE, THE AKALYSIS hoURD HOT a

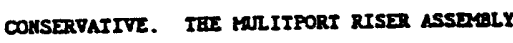
MTIBOUT ANY IMSTALLED EOUIFATIT SEDOTD IE CAPABLE OF WITHSTANDIMG THE GAS LOAD.

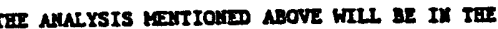
STRUCTURUL ANALYSIS RERoRT.

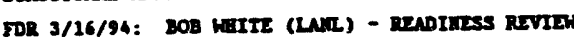

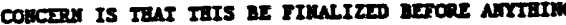
ELSE BE PUT arro TRE IAMR.

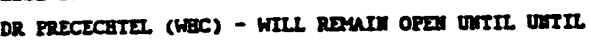
geport is Fimusizid.

7/15/94: IBE BOLD DOWA AICBOO BOLTS ARY SI2ED FCO

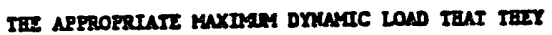

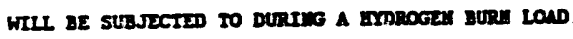

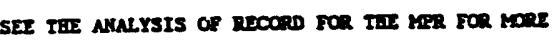

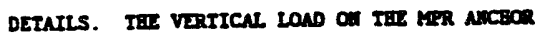
BOLIS IS CAUSTD BY TEI RRESSURE LOAD PLUS $N$

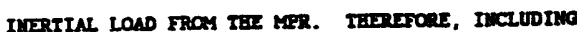
NI ESTIMATED MASS FOR IFE INSTALLD Cantontats Is Conservaitive.

smenson JP

I I RR A STRELON JP C

$07 / 25 / 94$ 
Paso : sto. 1

$08 / 01 / 94$

Hettront RISIR

SIMAL DESTG RETTEI

caserers Datuase

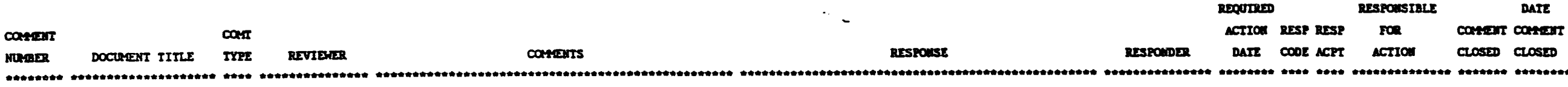

FDR00279 MELTIRORT FINAT DESTGS REVIEN:

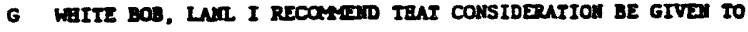
CBARACTERI2IMG RISERS SA AND SI FRTOR TO

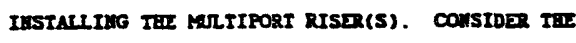
FOLLOWTM POINTS:

A) IF IEE RISER IS WAY OUT Of RLLES, IT WOUL BE USERUR TO RNOW IBIS EACT SO TRAT RISER UTILIZATIO

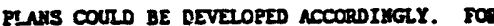

Tustance

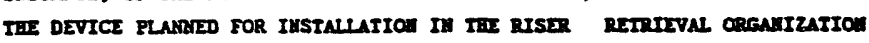

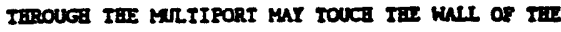

RISER. HITBOUI CBARUCTERHzIMG IEI EISER

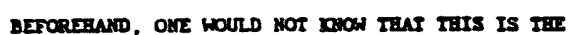

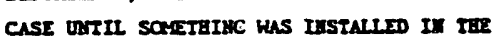

MeILITPCRT RISER AND THE DETICE KAS DAMACZD O

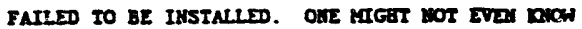

TEAT TIE SONIC PROBE, FOR IMSTANCE, uns TOUCBTMG IIE HNIL. AS DIRECT VIDEO OBSERVATIOUIS HOULD MOT

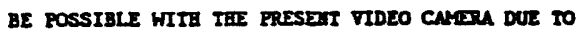

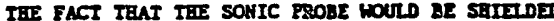

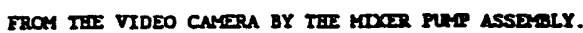
1) IF THE RISER IS SERTOUSLY OUT OF ROOND. IIIE HeIITRORT RISER MAY HOT FIT, AND IT WILL HIXD TO

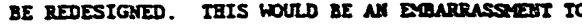
TBE PROTECT, AND COULD AFTECT THE SCBRDUTE. THIS

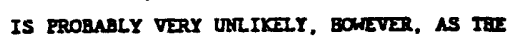

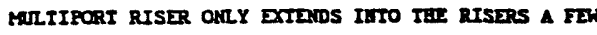
INCAES. AND IIE DESIGN IMCLUDES QUITE $A$ BIT OF

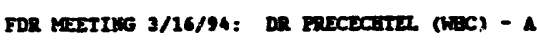

or ersecsearzer

I An

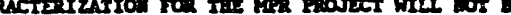

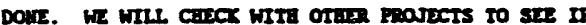

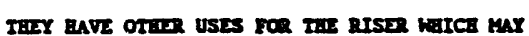

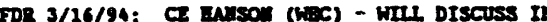

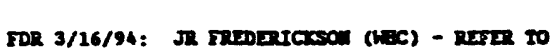

Response 0243. 
coreant Datnease

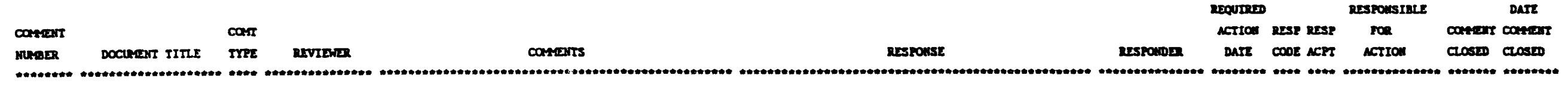

Tolornice.

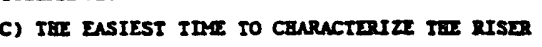

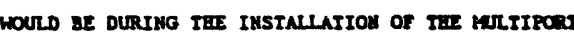

RISER WHEX TBE RISER IS OFEN.

D) TEE STATE OF THE RISER HOIT BE USETV

IMFORMUTION FOR RETRIEVAL PLMMTING. IT MAY BE

POSSIBLE TO GET FUNDING FOR RISER CBARCTTIRIZATIOW

ACIIVITIES ERTAY IEI REIRIEVNLL FrOCRNM.

hBILE I RENLIZE DOIMG THIMGS IS TII INAK PABA ARE

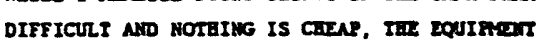

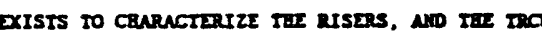

BAS BEEN SUCCESSFULLY USED OA RISER 12A. I

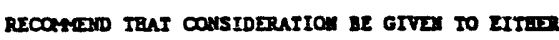

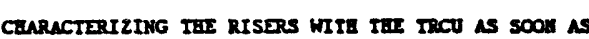

POSSIBLE OR DURIMG IEI INSTNLLTIOE OF III

MUITIRORI RISER(S).

TORoozeo MarTIPORT FIMAS DESIGA REVIEW:

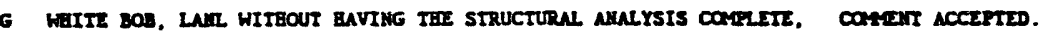

IT IS DIFFICULT IF NOT Imposstall to DetrpertaE Ir

IFE DESIG MESTS THE ACCEPTANCE CRITRLA.

RIIEASTING TBE DEVICE FOR FABRTCATIOH AI TEIS PONMT

ENTAILS RISKS IEAT TEE PROFECT MAY MOT WANT TO

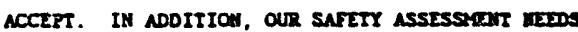

TEE RESULT Of TEE STRUCTURe Aratrsts to aE

COMPLETE. WE WTLL PROBAaLY Weatre TEE ITRST DRATI

OF THE SA WITB SOAE -TBD:" POR COArror LDITS AS

BEOUIRED UNTIL TER STRUCTURNL ARLYYSIS CAN BE

APPROVID. AND WE WTLL BATE TO RAV A DISCLADER IN
DR Frectearen

$03 / 16 / 94$ 
PeE: No. 3

$00 / 01 / 94$

MalTIRORT RISER

FIMAL DESIGN REVIEW

COAzart DATABASE

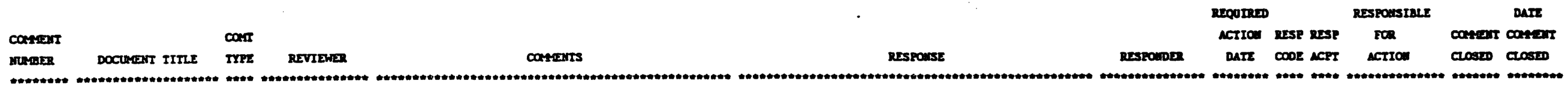

THE SA TBAT SAYS TEE SA IS COATIMGEAT OA A

SUCCESSFUR OUTCOAE OF THE STRUCTURAL MuRIYSTS.

EDR00281 GULTIRORT FIMAL

DESTGA REVIEN:

STRUCTURAL DESIGI

SPEC, BAGE 8, ITEY -

EARTR PRESSURE LOADS

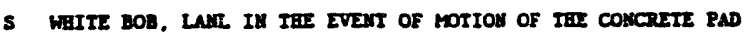
RELATTVE TO TEE RISER (POSSTALE DUE TO RROST EEAVES OR SETILING OF THE PAD) COULD IUTroOUCE

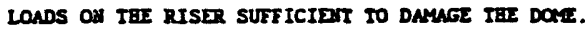
IN MY VIEW. DESIG FROVISIONS SBOURD BE MADE FOR

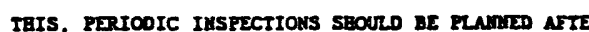

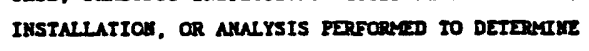

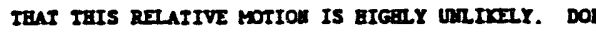
ORDER 6430.2A, SECTION 0111-3.4.1 STATES:

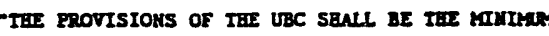
REQUIRDEENTS FOR FOUNDATIONS DESIGA. THE POTERTINL ADTERSE EFFECTS OF FROST EEATE AND COVDERTIS DUE TO EXPANSTVE SOILS SBALL ALSO DE COASIDERED III IBI DESIGK." "rOR ALL STRUCTURES. SECTIOM 0201. SURSURFACE INVESTIGATIONS, SQNLI BE COAPILID MITI TO DETDETINE SUBSURSACE CORDITIONS. RECOAIDDD FOUNDATION TYRE, ALLOUABLE DESTEM SOIL DEARING RRESSURE. SEISATC POTZMTIAL, AND DIFTEREMTIAL SETTLDMAT." SECTIOA 0201 STATES: SETILEAEMT AMALYSIS UNDER DIFFEREMTIN DESTG LONDS SBALL BE PERFOPMED MATRE DIFTEREMTIAL

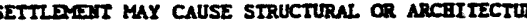
Damace.

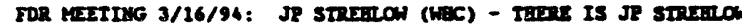

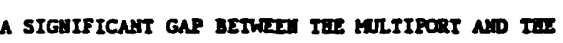

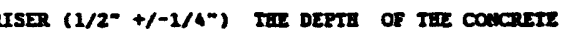

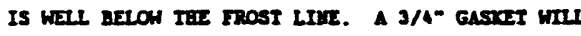

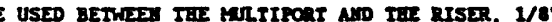

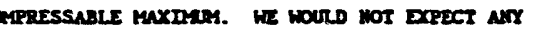

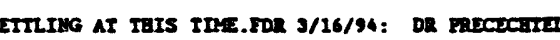

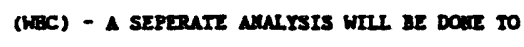

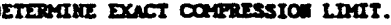

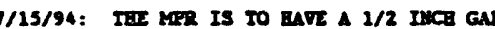

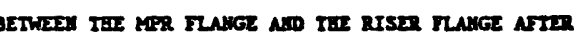

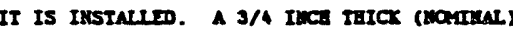

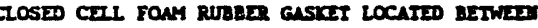

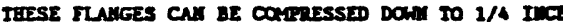

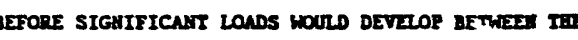

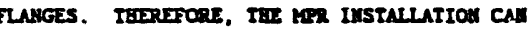

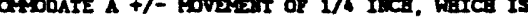

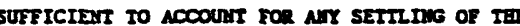

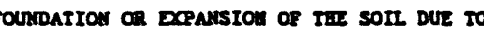

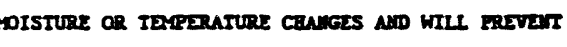

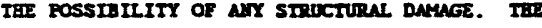
FOUNDATION IS LOCATED 3 FELI BELON GRADE. NEICE Is 6 IMCBES DEEPER TENII TEE DEPTI REQUTRSD BY SDC 4.1

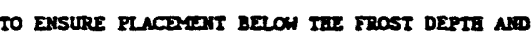


Pase Ho.

08/01/94 hertiport RisER

FIMAL DESTG: REVIEW

coresrr datasase

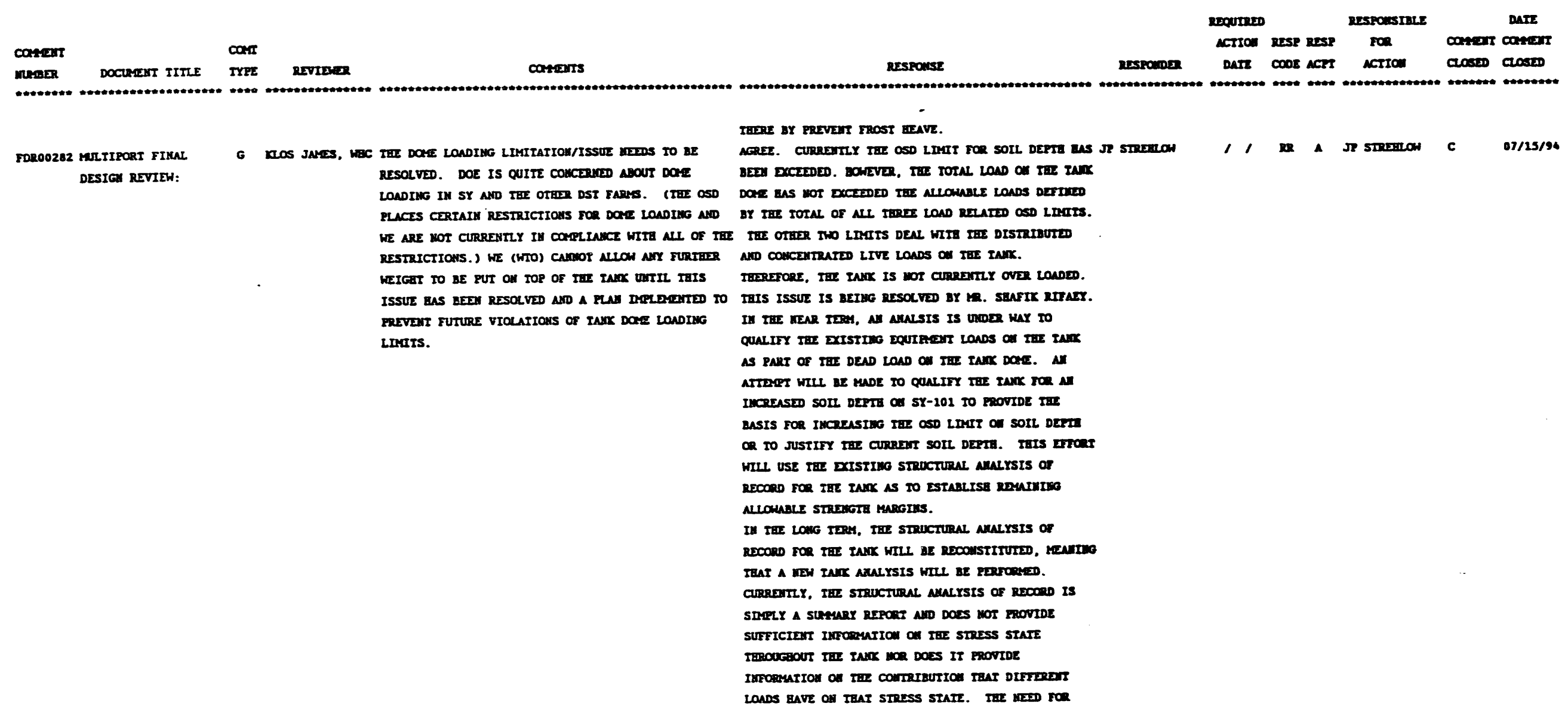


III

$\because 1$

:

直

章

尊安是

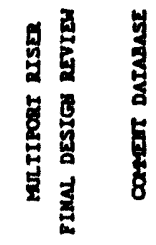

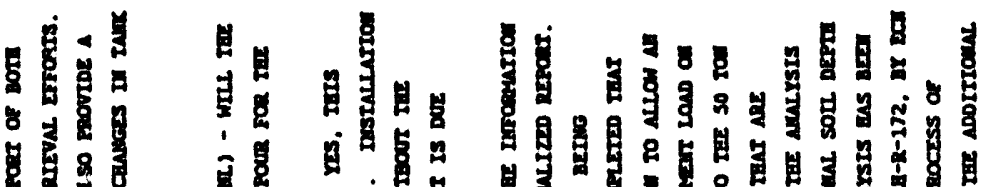

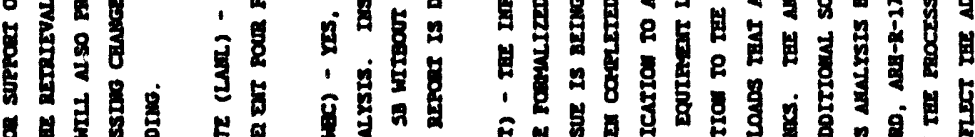

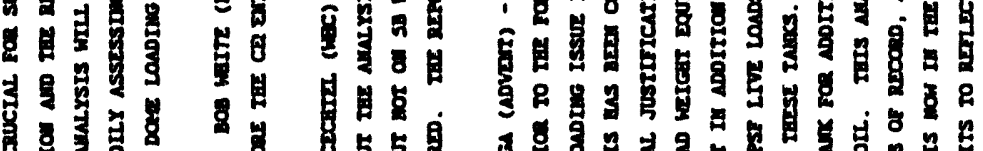

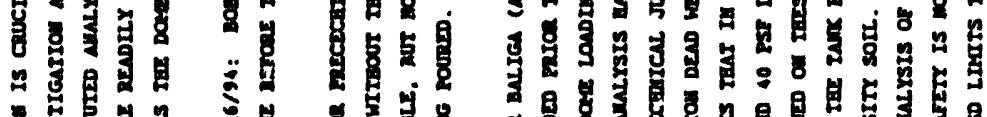

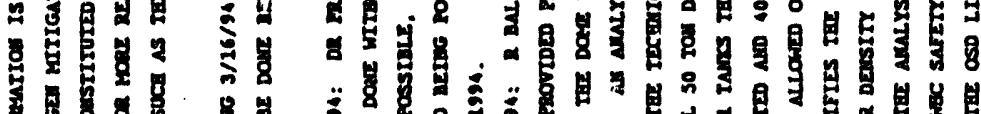

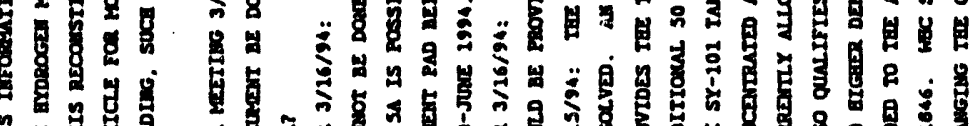

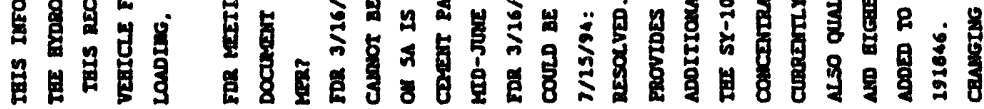


Pase No. $\quad$.

$08 / 01 / 94$

Merifiport RISER

FIUNL DESIGA REVIEW

carear dutasase

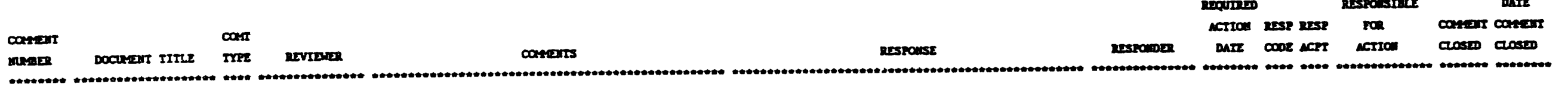

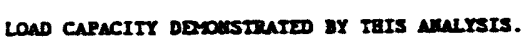

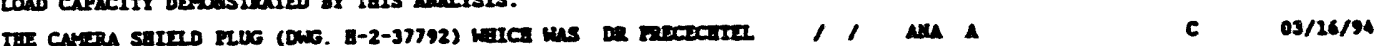

TDR00283 RETITPORT RISER

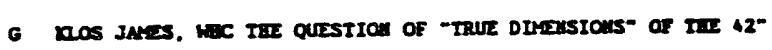

TIMAL DESIG REVIRH:

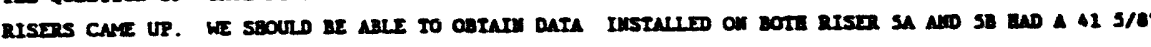

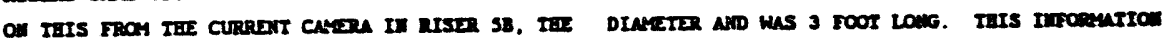

OC CA

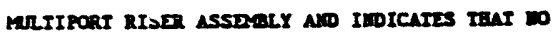

IMTERACE MITE TII A2- RISE WTLL OCCW

FDRoozes MerTIPORT FIMUL DESIGX REVIEN:

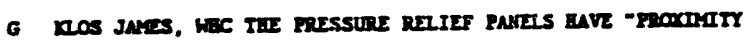

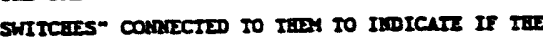

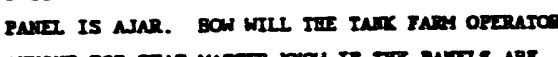

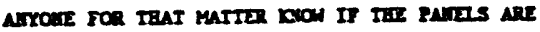

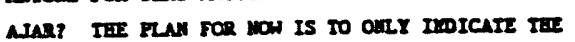

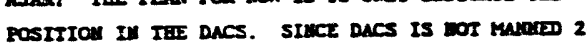

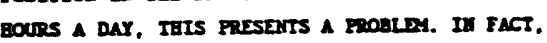

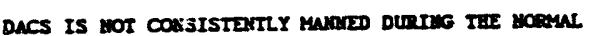
DAY-sarts BOURS.

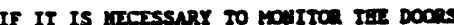

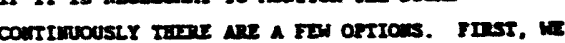

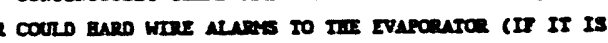

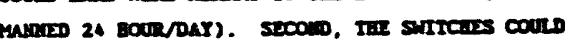

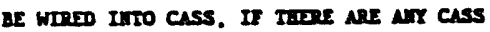

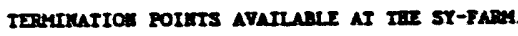

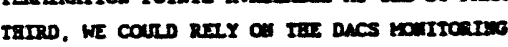

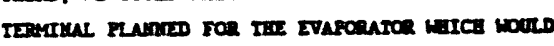
coremicate treouce III Genests sortwarr. BCWEVR. THIS UST NLTBentive worto at

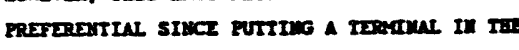
Evaporutor is atrenoy enet of till Dacs Full

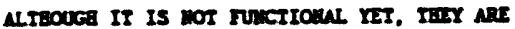
Hoaxruc on II.

FDR MEITING 3/16/94: Io Carstal (wac) -

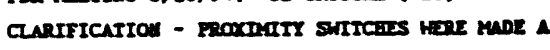

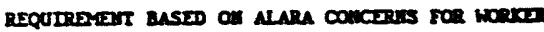
SAFETY orr OA THE IMTS.

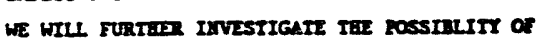

J Carsine

, 1

Is Corstal.

$07 / 25 / 94$ 
Pase No.

08/01/94

Muritiant erser

FIUNL DEster Revisa

casearr patasase

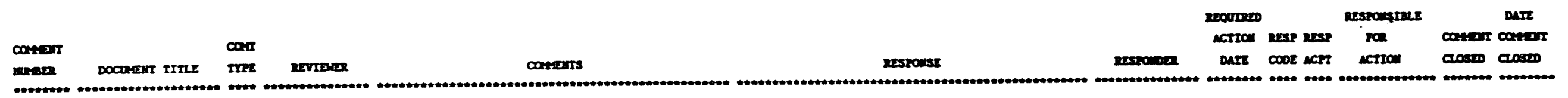

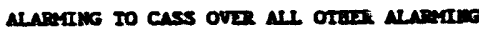

alterentives.

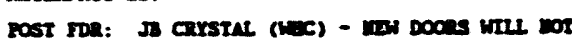

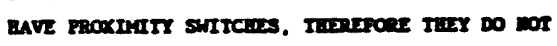

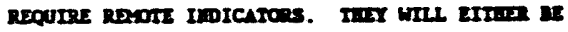

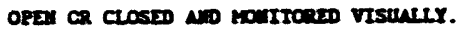

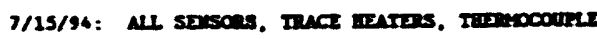

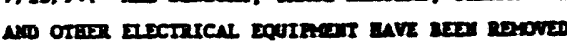

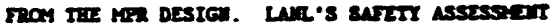

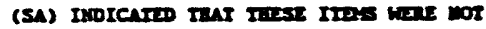

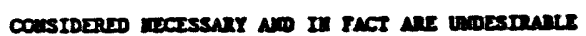

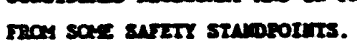

TDE00205 HETITORT FIML DESIGR REvizN:

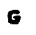

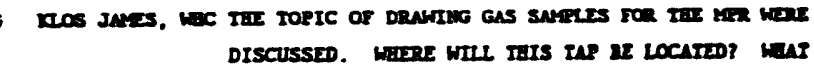

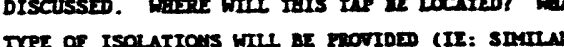

To III SES)?

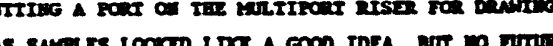

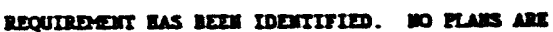

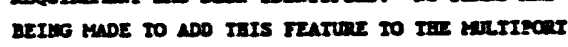

erser.

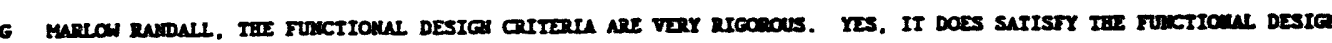

$\sec$

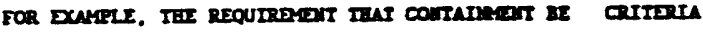

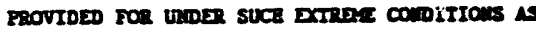

EARTBQUnTE, SHOW STORES. ETC. IS VERT STRICT.

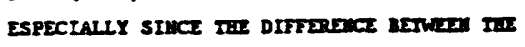

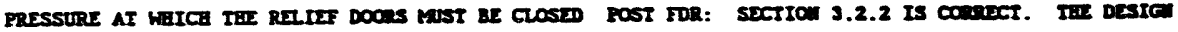

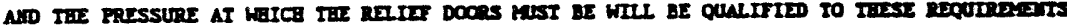

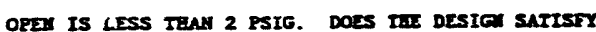

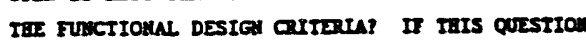

CRITERIA, SECTIOA 3.2 .2 on meromen
1, sen a

$03 / 16 / 94$
The 3/16/94: SII 25sposst 0290

smencen
$03 / 16 / 94$ 
Pese Xo.

08/01/94

Mattiport ristre

FINAL DESIGW REVIE

coneters Datasus:

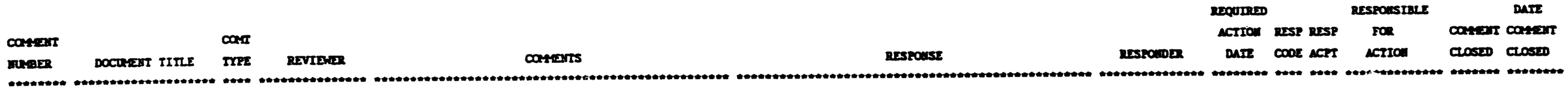

REMAIS TO BE AMSWERED, DOES A PLAN TOR OATAIHIMO

IBE AMSWER DXIST? IF TEE "FINUL DESIGA" DOES

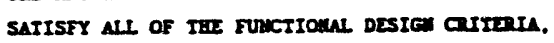

WTL II BE מDOTFIED?

50500587 histrost zinus DESIG REVIEN:

FUICTIOHAL DESTG

CRIIRRIA, SECTIOA

3.2 .2

FDR0028S MEIIIPORT FIMAL

DESTCa REVIEN:

TUICTIOANL DESIG

corteria

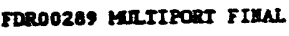

DESIGA REVIEN:

G MARLOH RAMOALL. WEDRE ARE IEE STRUCTURAL AKALYSES? $\angle$ DESIGX IS

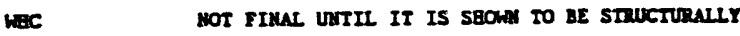
sound.

TEL XEFETED USE OF THE QUASTITY - 1.36 PSTOIs Tars TRere?

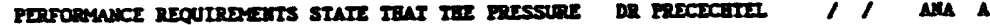

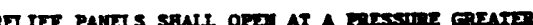

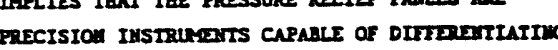
BETHEE PRESSURES WaICR VARX AY A RTIE 0.01 PSIG.

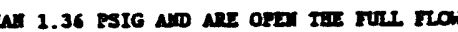

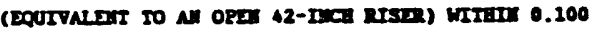

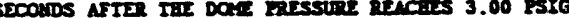

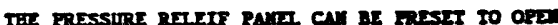

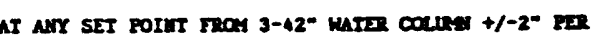

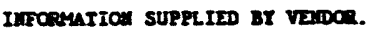

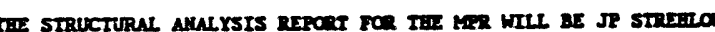

1 asa

$03 / 16 / 94$

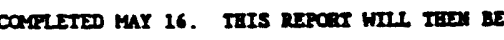

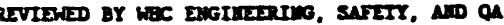

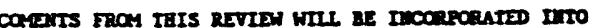

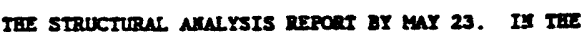

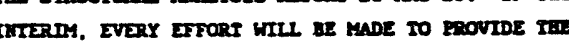

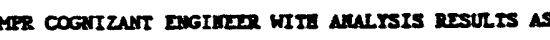

SHEY BECONE AVATCABLI.

CTRPETr

JP STREma 
Pase Ho. 9

$08 / 01 / 94$

artitrort grsen

FIMAL DESIGX RETIEN

coneart Dataress

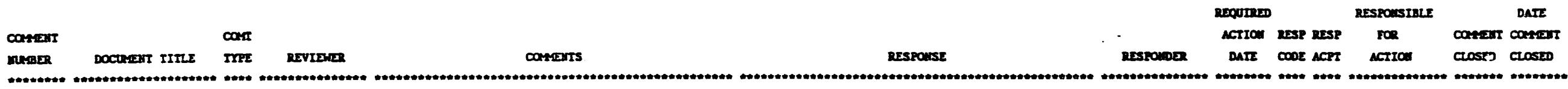

DESIGE REVIEN:

Bnzuros

$\operatorname{mac}$

IDEMIIFICATIOA

SDR00291 MUITIPORT FIMAL DESIGA REVIEN:

anzNeDS

IDEMTIFICATION
ENTITLDD TELIIPORT BAZARDS IDEMTIFICASIOW" ARE VAGUE AND OFEN-ENDED. FOR DDMPLL, OAE CA"SE OF

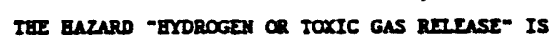
DESCRIBEd As "ETTERMAL FORCLS OR EVEMTS". hant DOES THIS MENN? IN ANOTHER DCATPLE. "UNEXPECTED

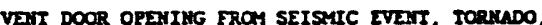

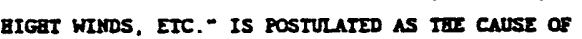
THE BAZARD "UNFILTIRED RELEASE TBROUGA OFEN

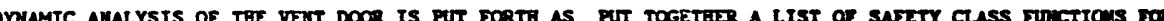

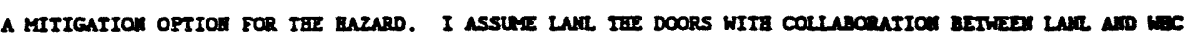

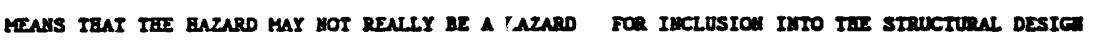
gICAUSE A DVANAC ARALYSIS WILL SBOW TBAT

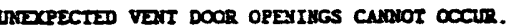

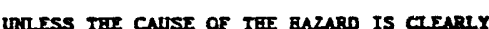
SPECIFIED, HO SUCB AKNLYSIS CAM BE RROOUCED.

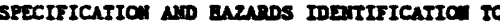

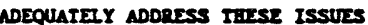

7/15/94: SAFETY CASS Functions bAVE Bes

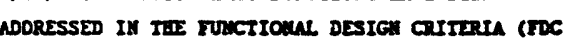
AND III TEE LAMI SATETY ASSESSEDarT (SA), RET 20.

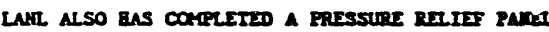

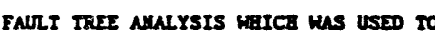

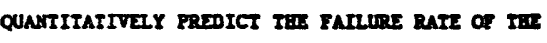

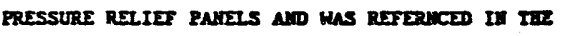

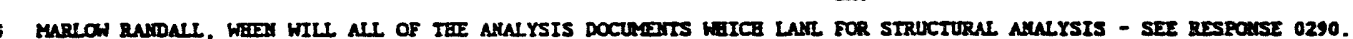
WEC REQUTRES BE PROOUCED? IF AN AKULYSIS SBOWS IBAT THE BAZARD IS GENUINE. WTLI THE DESIGA BE WOTFIrD? FINISBED. IT WILL DE ISSUID AS A SURPORTTEO

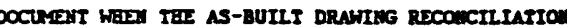

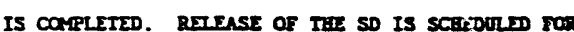
Aucusi 5. 1994.

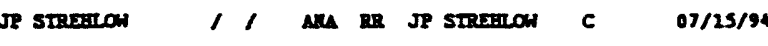




\section{sase to.}

00/02/94

hertiront niser

rinc desion arview

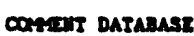

\begin{tabular}{|c|c|c|c|c|c|c|c|c|c|c|c|c|}
\hline $\begin{array}{l}\text { coments } \\
\text { masen }\end{array}$ & Docunterr IITX & TYRT & revienas & conemers & Mestowse & messomen & $\begin{array}{l}\text { neoutand } \\
\text { action } \\
\text { Dart }\end{array}$ & & extert & $\begin{array}{l}\text { mesponsiats } \\
\text { row } \\
\text { nction }\end{array}$ & $\begin{array}{l}\text { conement } \\
\text { closem }\end{array}$ & $\begin{array}{l}\text { Dure } \\
\text { cancost } \\
\text { crosers }\end{array}$ \\
\hline ร28200392 & 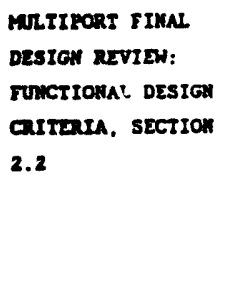 & $\sigma$ & $\begin{array}{l}\text { MTLLR IILL, } \\
\text { LNR }\end{array}$ & 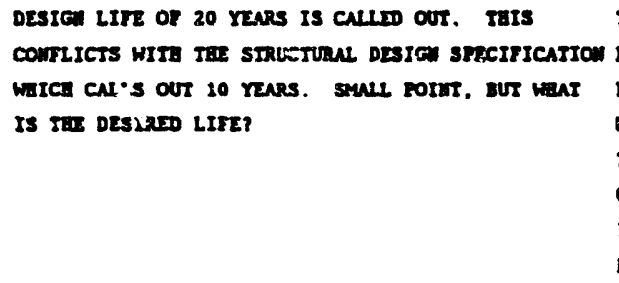 & 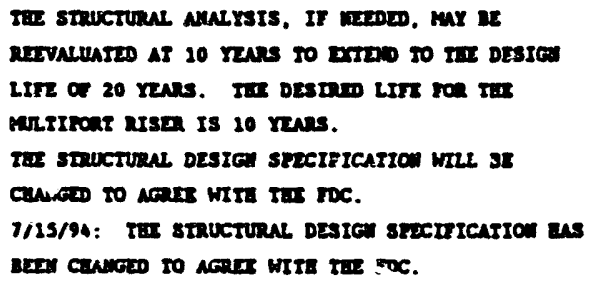 & on mrexedrs & 11 & I & $A$ & JP smench & c & $07 / 25 / 94$ \\
\hline roR00293 & 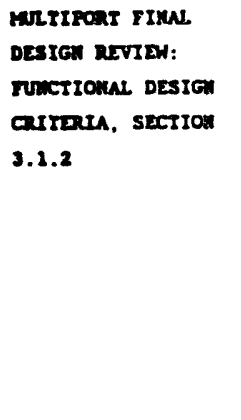 & - & $\begin{array}{l}\text { MrLun arf. } \\
\text { LnR. }\end{array}$ & 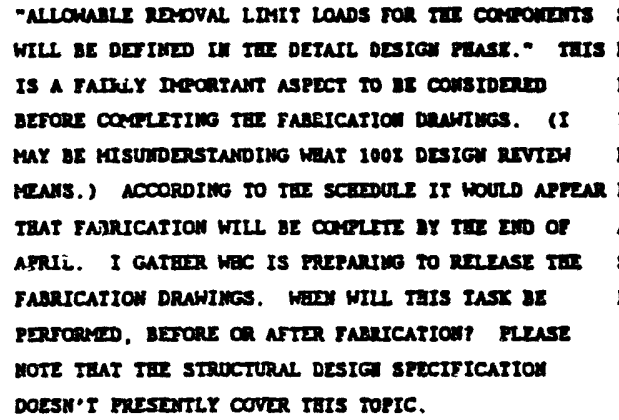 & 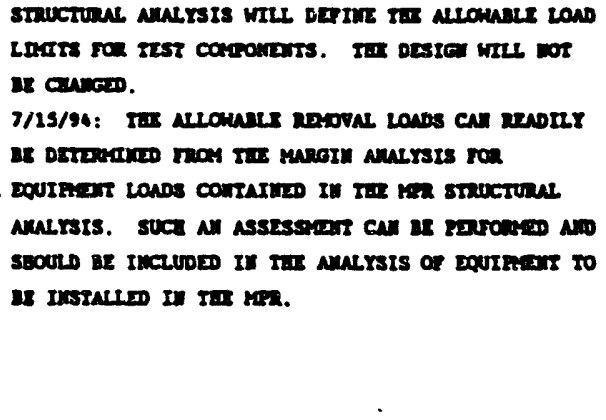 & 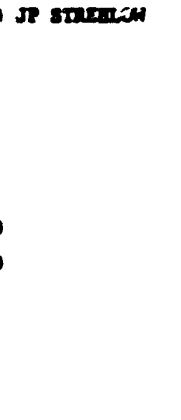 & 11 & $\mathbf{m}$ & a & Je smanton & c & $07 / 25 / 94$ \\
\hline rOR00294 & 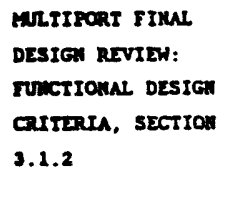 & 0 & $\begin{array}{l}\text { Mrung BrL. } \\
\text { LNII }\end{array}$ & 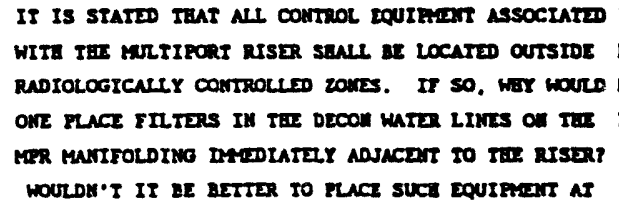 & 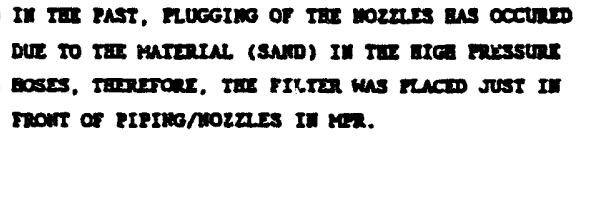 & De rexesering & 11 & nean & $\boldsymbol{A}$ & & c & $03 / 16 / 94$ \\
\hline
\end{tabular}


Pace xo. 11

0es/01/94

Hertiroat RISER

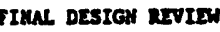

careart datagase

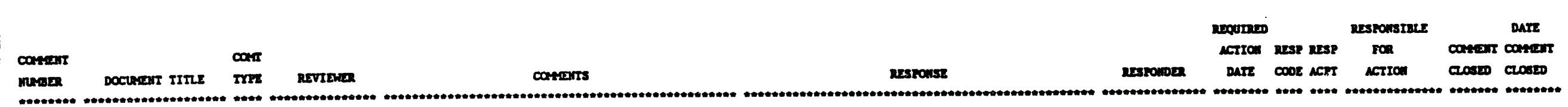

FDRO029S GerLITORT FIMAL

DESTEM REVIEN

TUICTIOARL DESIG:

crirenia, section

3.1 .2

Togoozg6 hertroort FIMN DEsien REvisw: DESIG DRANTMGS

SEction 5.5.1

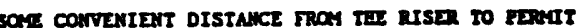

EASIER ACCESS AND SERTICrimot

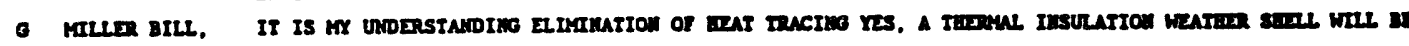
LMR. IS AEIKG SUPPORTED BY TIE ASSERTtOW THAT TEE BLCED over IIII rRotzctive catos OutsIDE III

on mercestr

1,2

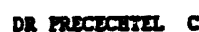

$07 / 15 / 9$

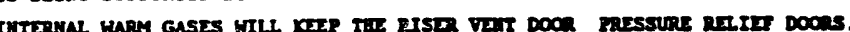

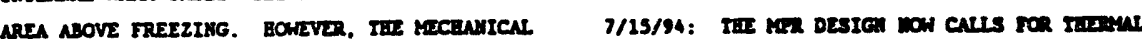

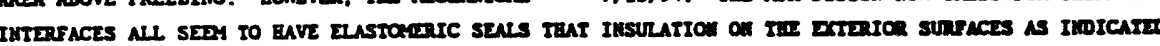

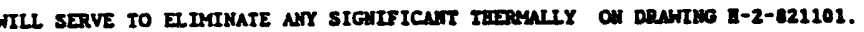

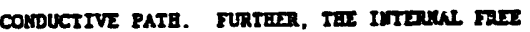

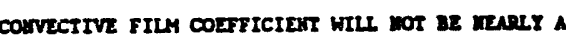

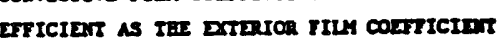

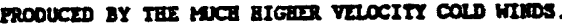

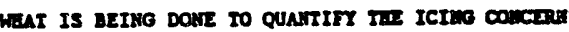

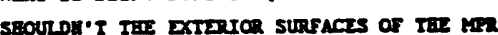

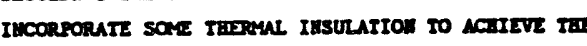

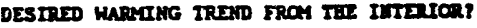

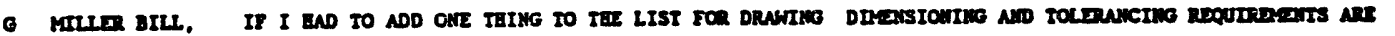
LAR.

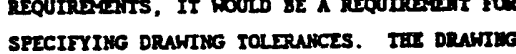

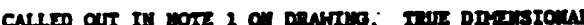

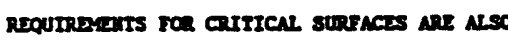

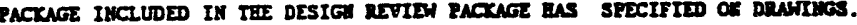

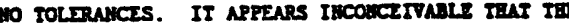

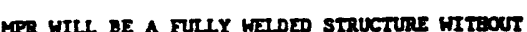

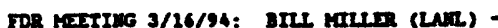

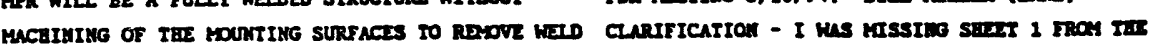

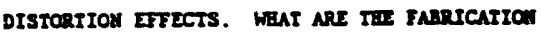
destew reviza rackere.

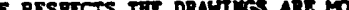

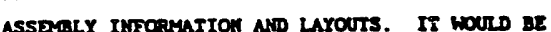

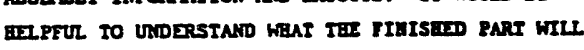

BE LINE, AND HITE TBIS INTORMATIOA SORE 
Pase No. 12

00/01/96 entroos giszo

FIMNL DESIG REVIEN

corsedr DATABASE

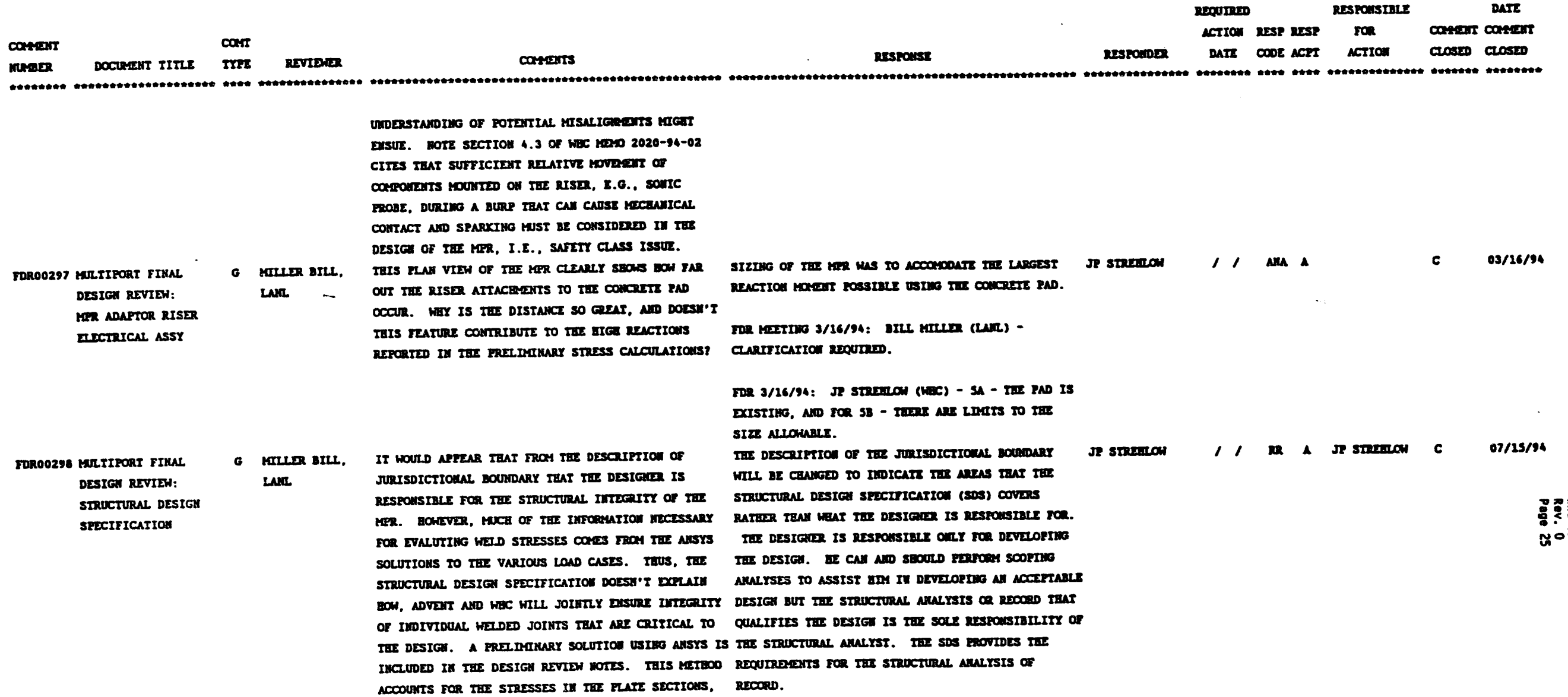


Base ทั. 23

$08 / 01 / 94$

MULTIPORT RISER

FIUNL DESIGA REVTEN

COAYIXNT DATABASE

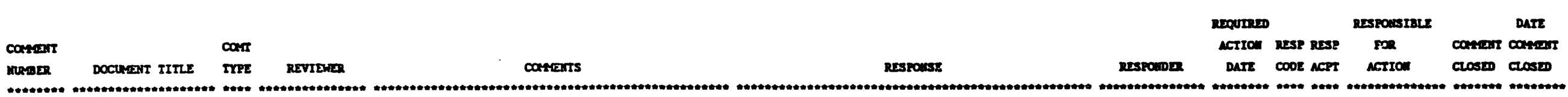

DOROO29, MartIRORT SIMAL

DESIGA REVIEW:

STRUCTURAL DESIGA

SEECIFICATIOH

SDR00300 EULTIPORI FIMAL

DESIGW REVIEW:

STRUCTURAL DESIG

SPECIFICATIOA

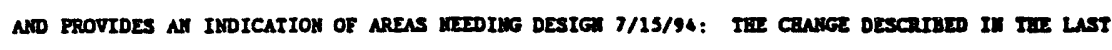

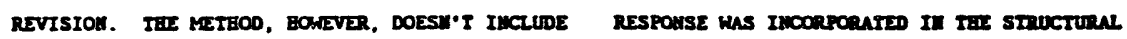

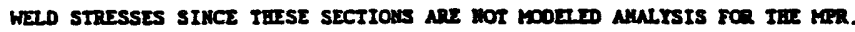

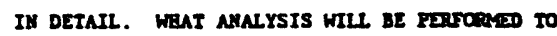
ENSURE WO STRUCTURAL, FAILURE WIIL COCOR IN IRE WZD SECTIONS, CAUSIMG STRUCTURA FAITURE III GRE ANO BURN EVENT? BOH WILL TEE IMFORMATION OE DOCUNENTED?

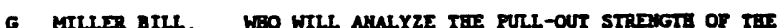
Land

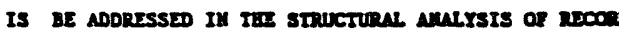

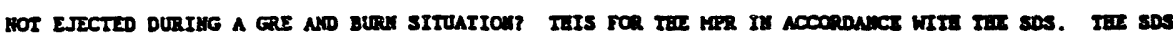

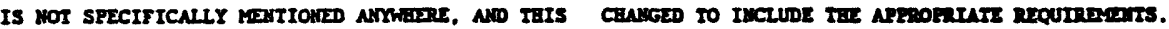
EDTENT IS IDENTIFIED AS SASETY CLASS 1.

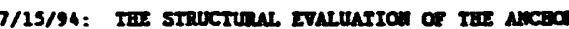

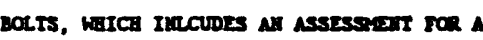

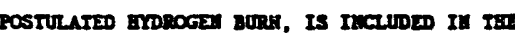

STRUCTURNL NMLYSIS TOR TEE HER.

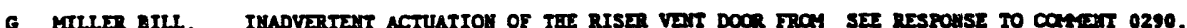

UNUSUAL EVERTS SBOUTD BE ASSESSED AN MTITGATED IX

UNUSUAL EVZHTS SBOUID BE ASSESSED AD HITIOAT

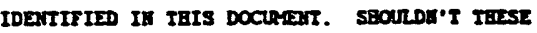

CAICORATIOHS BE MAOE PART OF THIS ETRORI? III

DOTNG SO, ONE MTERT COHSTDER TII COURLING ETrEet

OF OTHIR OPERATIMG SYSTES Ox IBE Vart DOCR

RESTRAINT SYSTEY. FOR DCNAPLE, WILL TEI SOUTC

PROBE EXCTIATION FREQUENCY COUELI WTTE TII MATRRAL

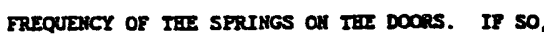

hOUTD TEI AMPLITUDE BE SUTFICTENT to CNUSE TBE

DOOR TO REIEASE?

JP smanon

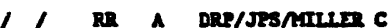

$07 / 25 / 94$

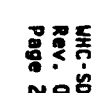


Pase No. 16

08/01/94

Eultikceat giser

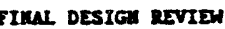

conerst Dasnense

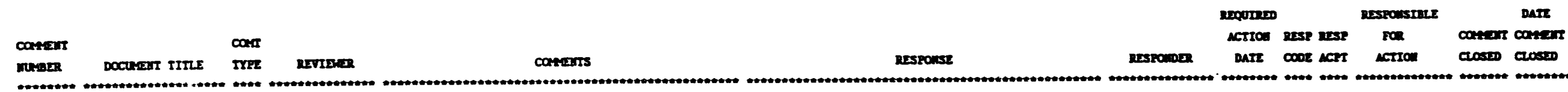

SOR00301 Eertiroer FIMAL Desian Revisu:

SRRUCTURAL DESTGN SPECTFICATION.

SEctrow 4.1

\section{1}

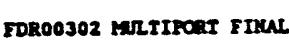
Desica sevisi: 8-2-821100, REv. 0 . DETAII Z. PAGE 3

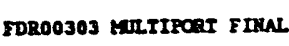
DESICX REVIDN: E-2-821100. REV.

TOROO304 ERTIPCRT FIMAL DESIGA REVIEN:

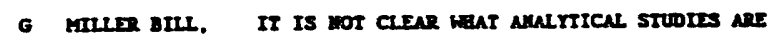

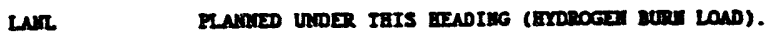

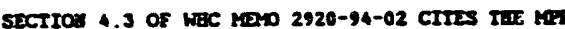

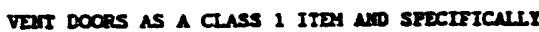

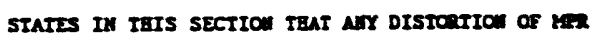

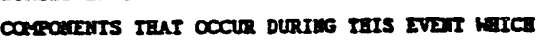

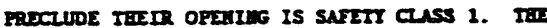

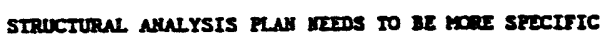
III TIIS RDEan.

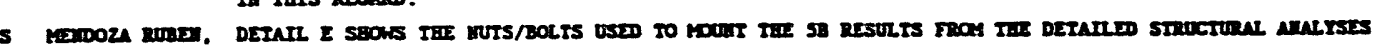
wac EULITPCRT TO THE 42- RISER AND WOTES -14

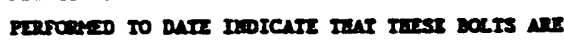

SEE RESPONSE TO Caregrit 0290.

Den erececarm

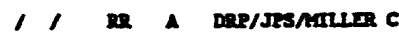

$07 / 15 / 94$

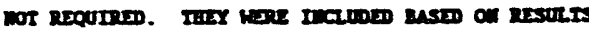

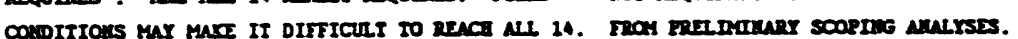

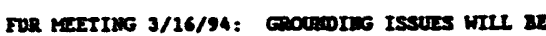

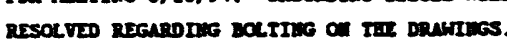

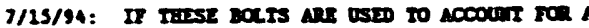

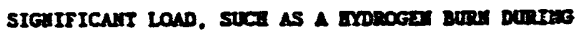

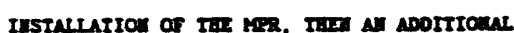

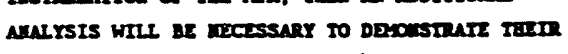

ABILITY TO RESIST SUCA A LOAD.

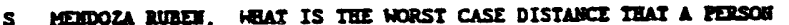

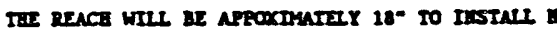

J smmon

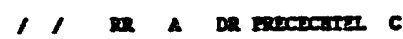

$07 / 15 / 84$

$\operatorname{mic}$

WOUID BAVE TO REACB TO PLACE A WUS OA COE OF TIS

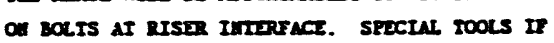

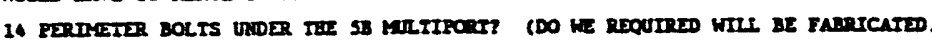

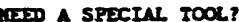

5 moza sures. WAC

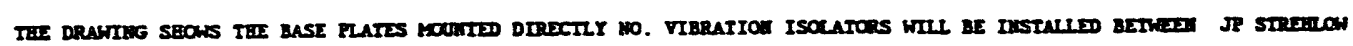


Pase Mo. 15

$00 / 01 / 94$

MeVITIFORT RISER

FINRL DESIG REVIEN

COEIENT DATABASE

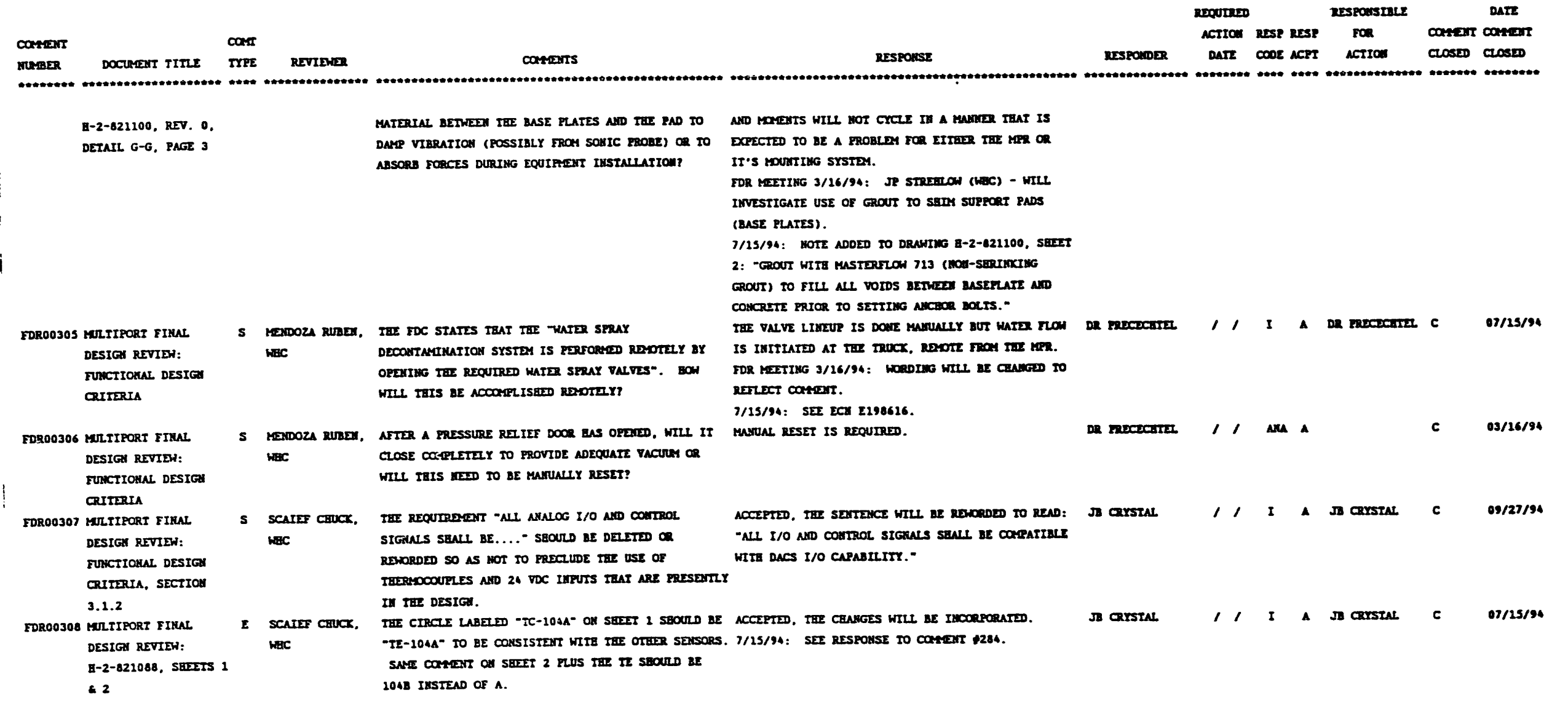


Page No. 16

00/01/94

MULTIPORT RISER

IIMAL DESIGA REVIEN

COMERT DATABASE

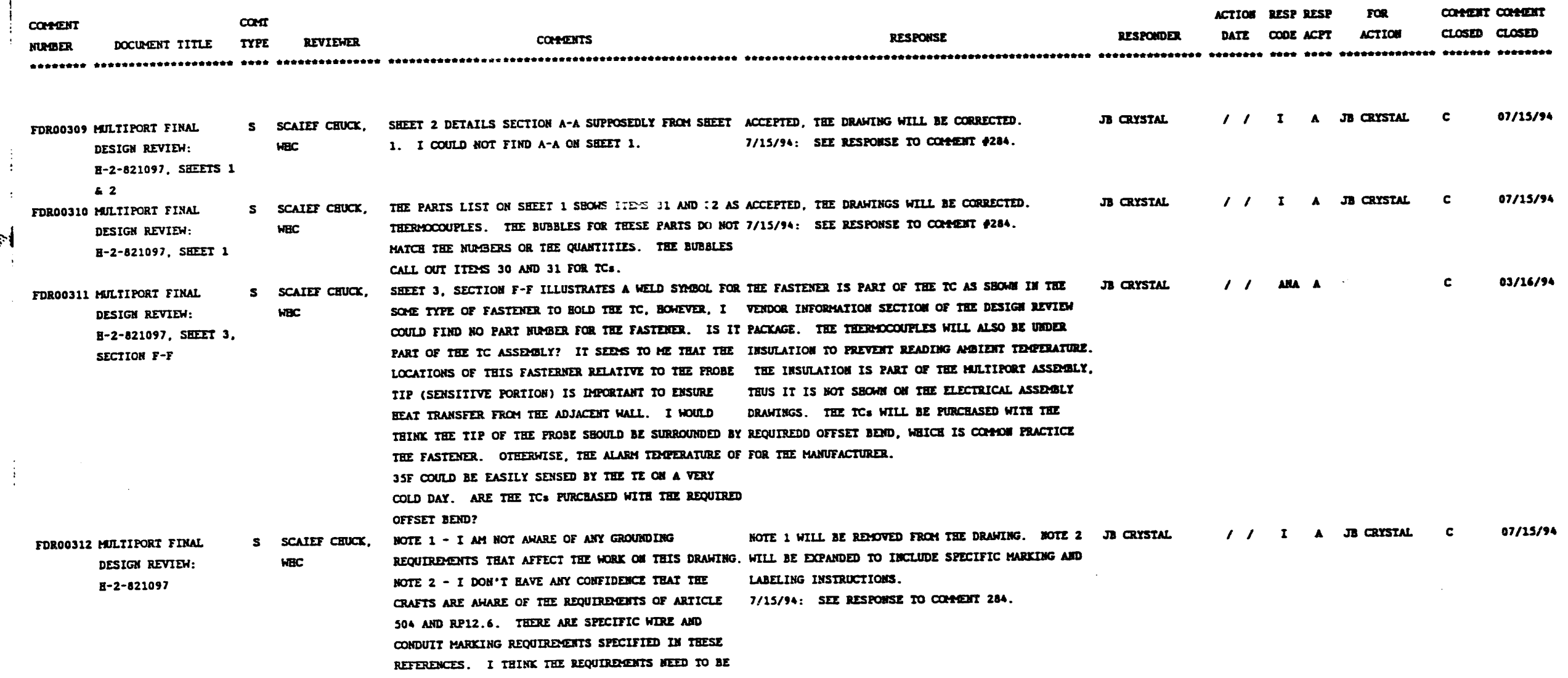


Pase Ho.

$00 / 01 / 96$

Fertiport RISER

FINAL DESTGW REVTEN

COREart DATABusE

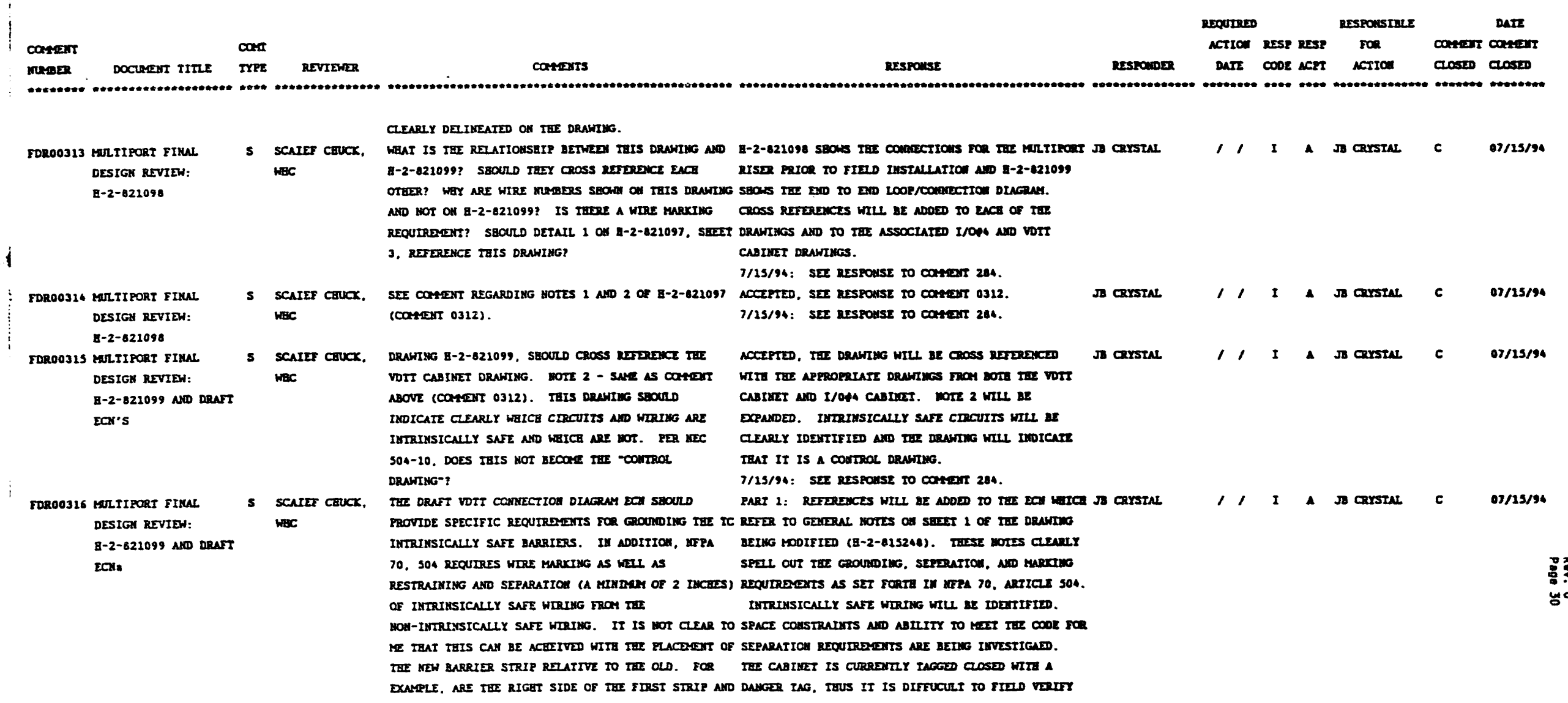


Pase Mo. 18

$00 / 01 / 94$

mentipons nesse

FIRL DESTG REVIR

conears Datashse

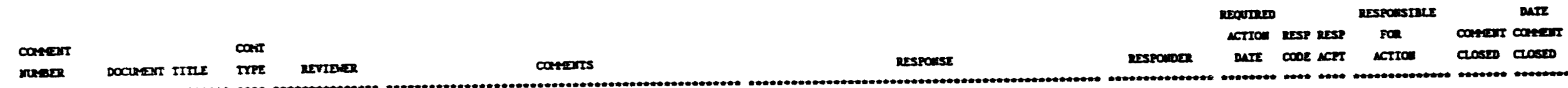

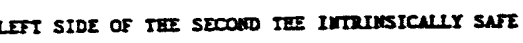

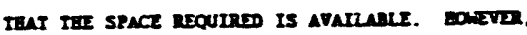

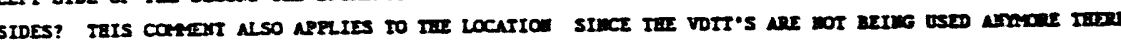

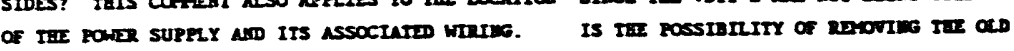

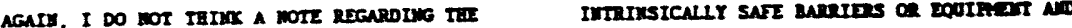

ape

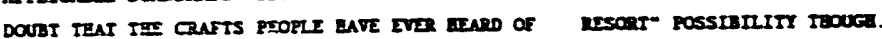

ISA RP12.6.

Irromenstion in and in

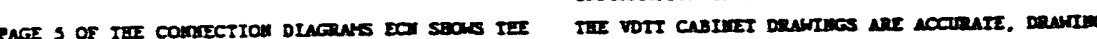

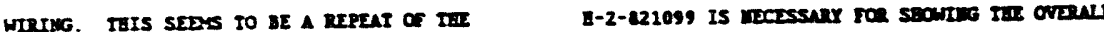

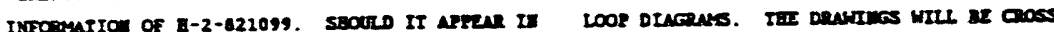

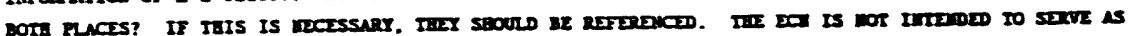

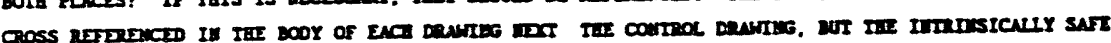

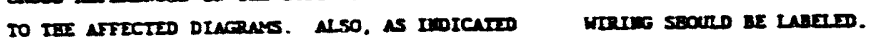

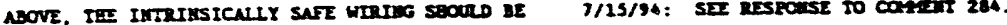

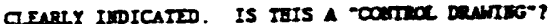

FDeo0317 MelltiFort FINAS DESIOA REVIEN:

merer mine.

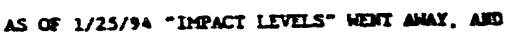

carear nocretso.

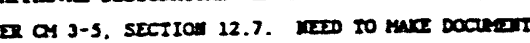

carererr.

Sectios 2.4

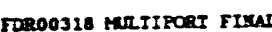

DESIOA REVIDN:

G meror max.

HORY PLAN. PACE 20.

Sxition 3.4

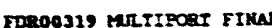

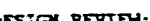
Fracticins desien

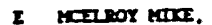

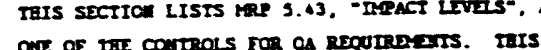

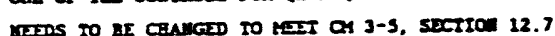

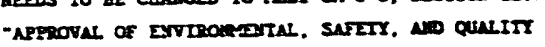

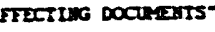

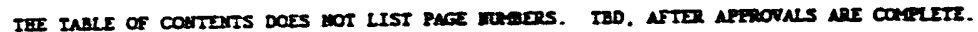

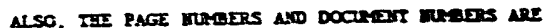

NOT LISTED OA TIE IMDTVIDUAL PAOES.

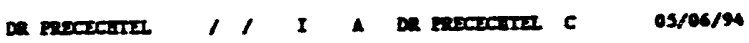


Rase $10 . \quad 19$

08/01/94

entroont ersa

FIRAL DESICA REVIR

congers Datasuse

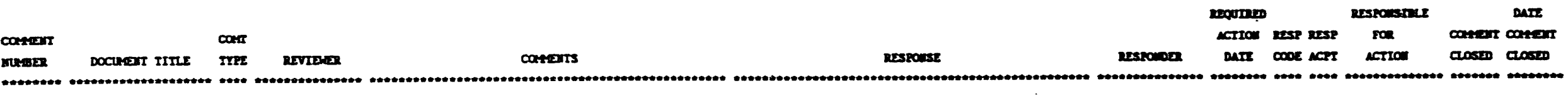

carteris

FDRo0320 EALTIPORT FIMAS

DESICA REVIEN:

InSTRuREarr LIST

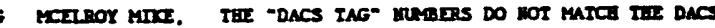

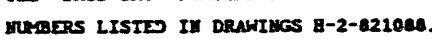

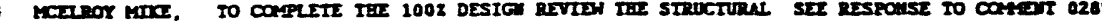
AMALYSIS gEPORTS MUST BE COATITID.

TDR00321 BellTIPORT FTWN DESICN REVIEN: $\operatorname{mac}$

STRUCTURA ARecrsis

semet

SDR00323 ERITIPORT FIMUL DESIGA REVIRN:

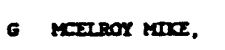
$\operatorname{mac}$

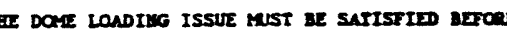

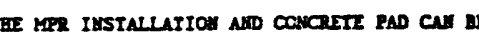
PuCED.

FDR00323 LARTIPORT FIKAT DESTGM REVIEN: Sugationil desice carteres

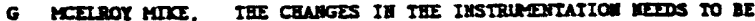
$\operatorname{tac}$ INCOP position ImICATORS. ETC.

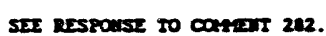

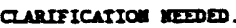

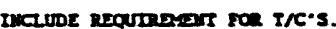

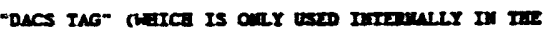

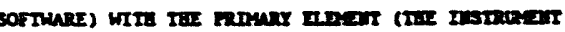

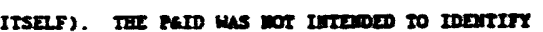

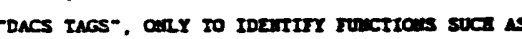

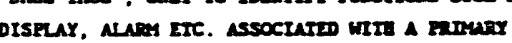
DDERT TBAT APREN II IIII DACS. IIII I/O LIST Is

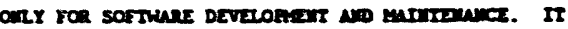

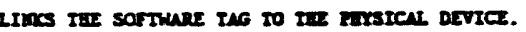

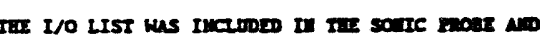

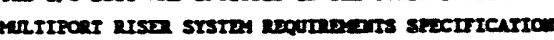

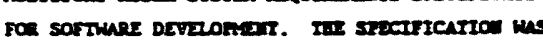

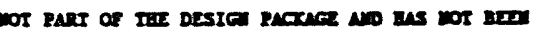
OFFICLACLY Rn resed.

1/15/94: SEE exspouse to carear 224.
1,1

$03 / 16 / 94$

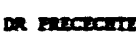

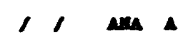

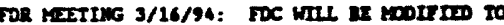

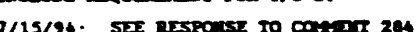

II sthe an

1, an 1

on Excrestrl 
Pase to. 20

$003 / 02 / 94$

Martiporis arser

FIMNL desich Revio

coreart ontnouse

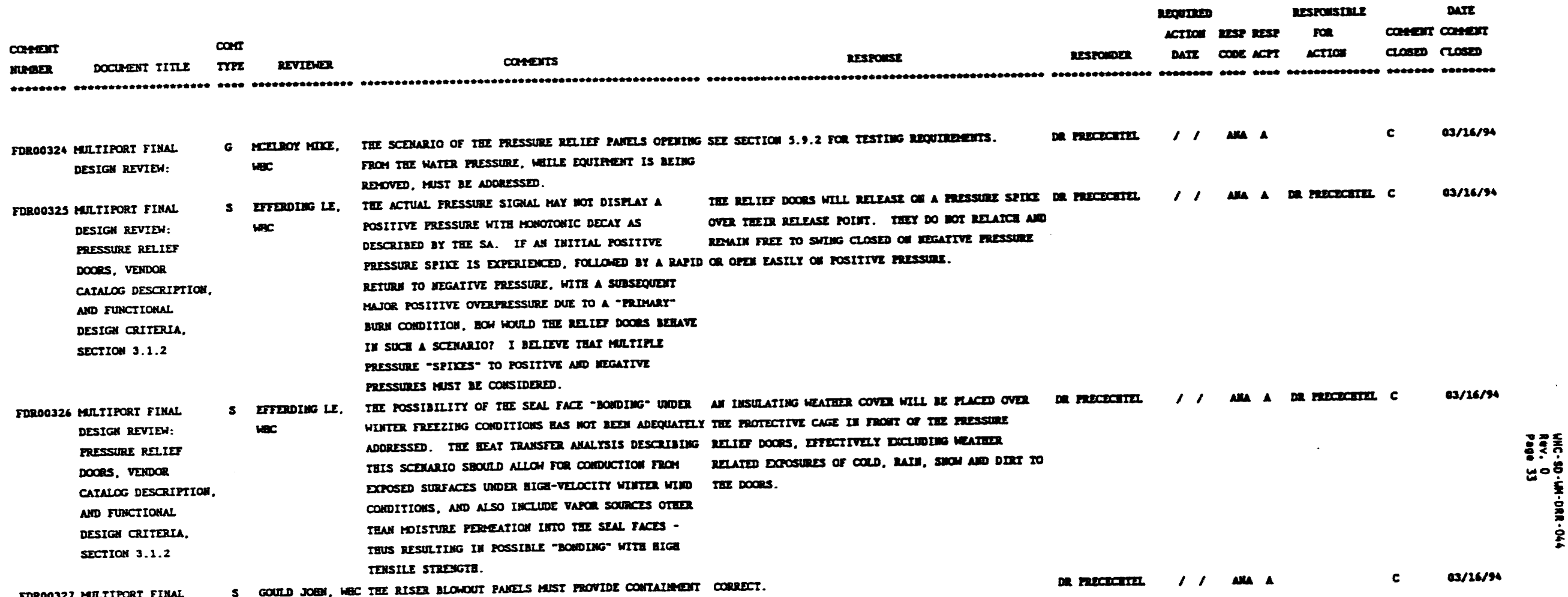

TORO0327 EURTIPORT FIMAS. DEstGa REvis:

ECA 198604. BLOCX

12. SECTIONS 1.3 .2

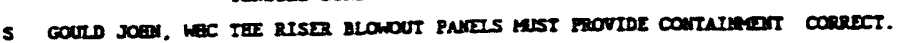

De rearaman

everse

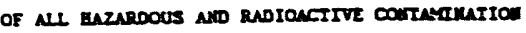

AND 2.3

WITHI IEII INAS. DCCIPI UMDER TII 
Pase to. 21

$08 / 01 / 94$

mintipoet eisen

FIn DESICU REVTD

corsent Dathense

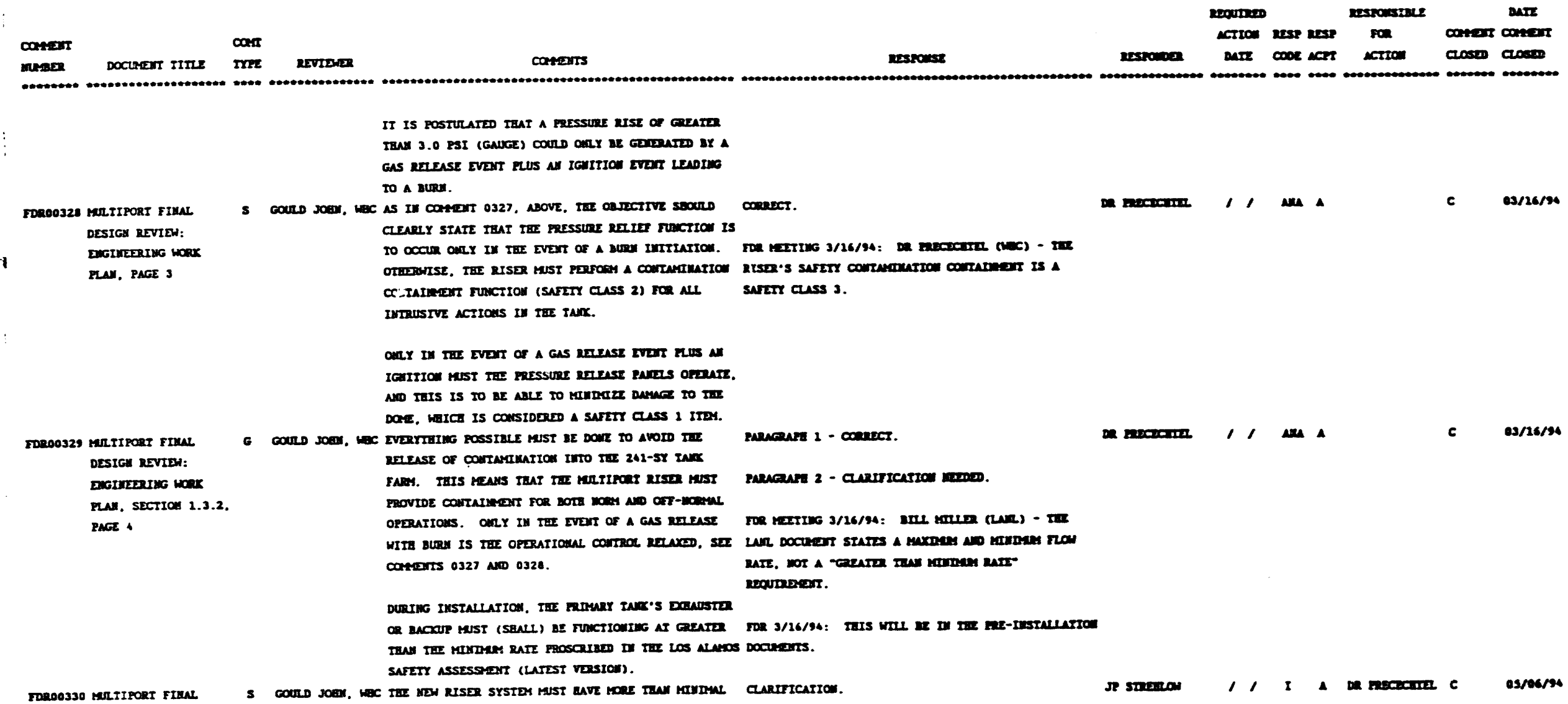


Pase Ho. 22

$08101 / 96$

artiport nise

Frus desiga revida

coneser ournenst

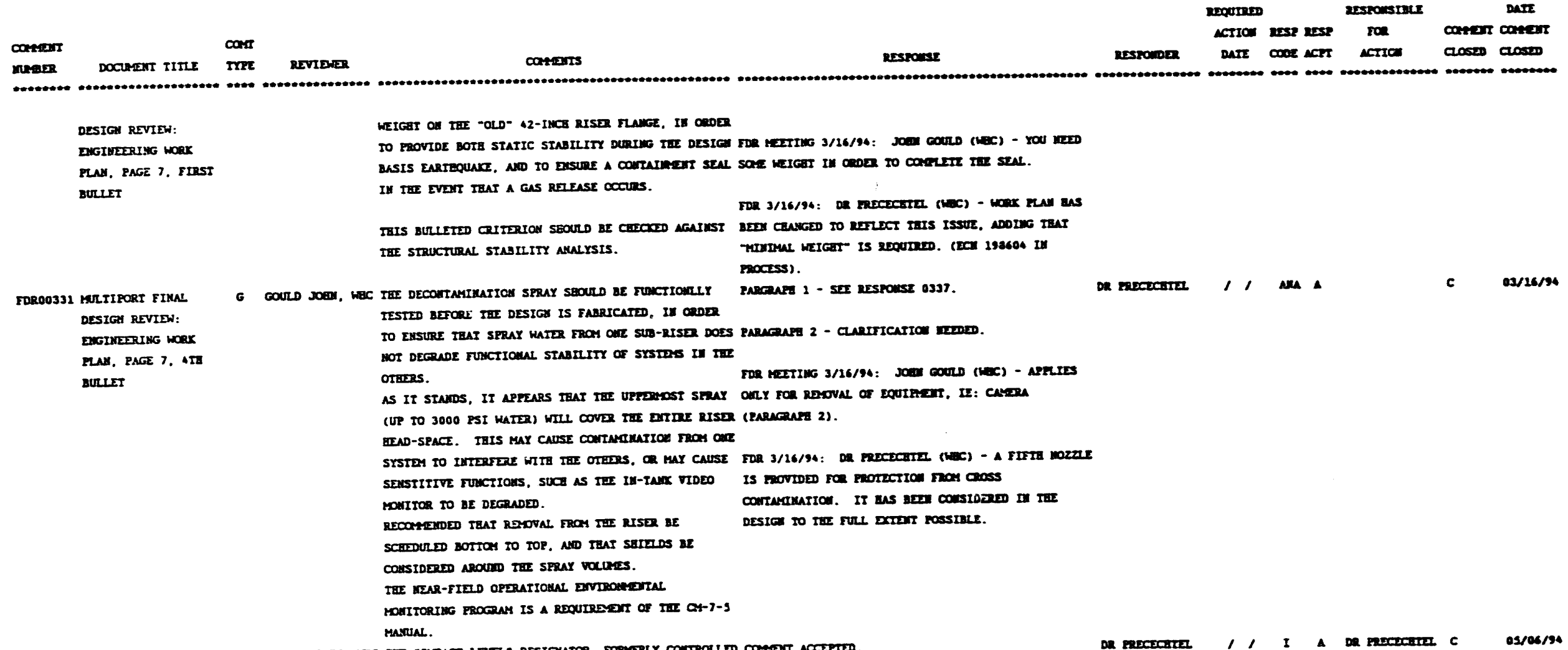

TOR00332 LALTIPORT FINAL DESIGA REVIIN: EMGIMISRING WORK

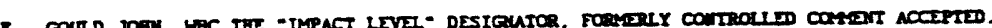

aY

Ca-3-3. SEction 12.3. 
Pase Mo. 23

$08 / 01 / 94$

sertipori rise

FIMAL DESIGX REVIEW

carterr DATABASE

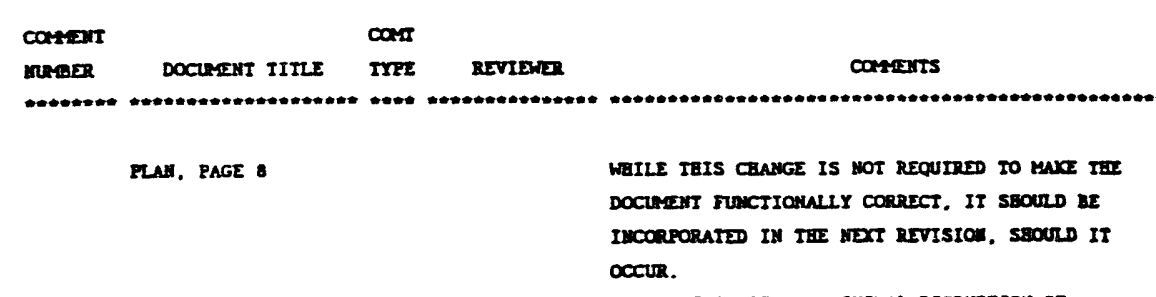

TDR00333 LELTIPORT EIMAI DESIGA REVIEW:

If TUMCTIONAL DESTE CRITRIA, SECTIOA

2.3. BUIEI A

FORO0334 EeTIIPORT FIMAL DESICA REVIEW:

FURCTIONAL DESTG:

CRITERTA, SECTIOA

3.1.1. BULIET ।

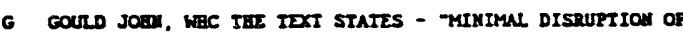

COATAMINATED SOIL.-

THIRE SBOULD $8 E$ A PRE-INSTNLATIOU (BASELIME)

SURVEY. CONSISTENT HITE MNNUNL WBC-CY-7-S. SECTIO

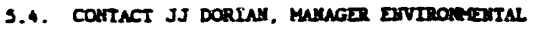

EMGTIEDRING STUDIES, TO COORDIXATE THIS REOUEST.

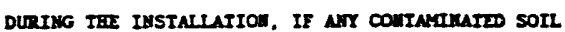

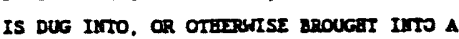

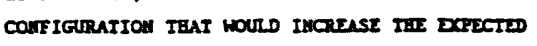

DOSE FOR HORCORS, IT SBOULO BE REPOVDD, SUITABLY

PACXCED AND DISFOSITIONZD IIS AOCORDAMCE HTTB

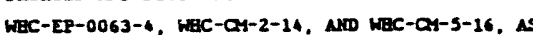
APPLICAaLs.

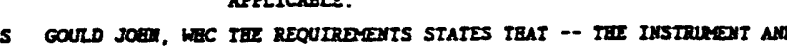

\section{COrTRCL(S) SENLI -COrTROL III Row OF}

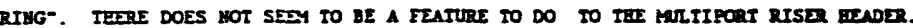

TBIS HITB DXACT CAPASILITIES.

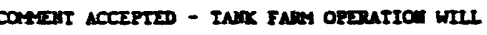

THIS PEOUIRTERTI

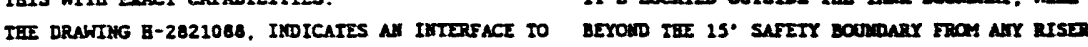

III SY-101 DECON TRUCK, BUT IT DOES OTHIBMTSE

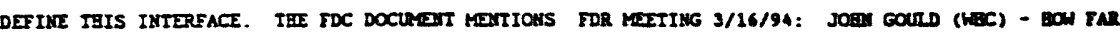

THE IRUCX, BUT NOT IEII OTHER IMTERYACT

reoungengers.

NHYY Fros RISER?

excuned usspoustales

natrou ess pesp tor

Dare

Date COOE ACIT ACTIO

corest coresen

IF IEE TRUCX IS TO BE OPERATED WITI DEGINE

FDR 3/16/94: DR PRECICBIII (WBC) - ABOUT $70^{\circ}$ -

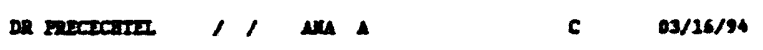


$00101 / 94$

mertifont nista

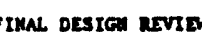

careart Datamst

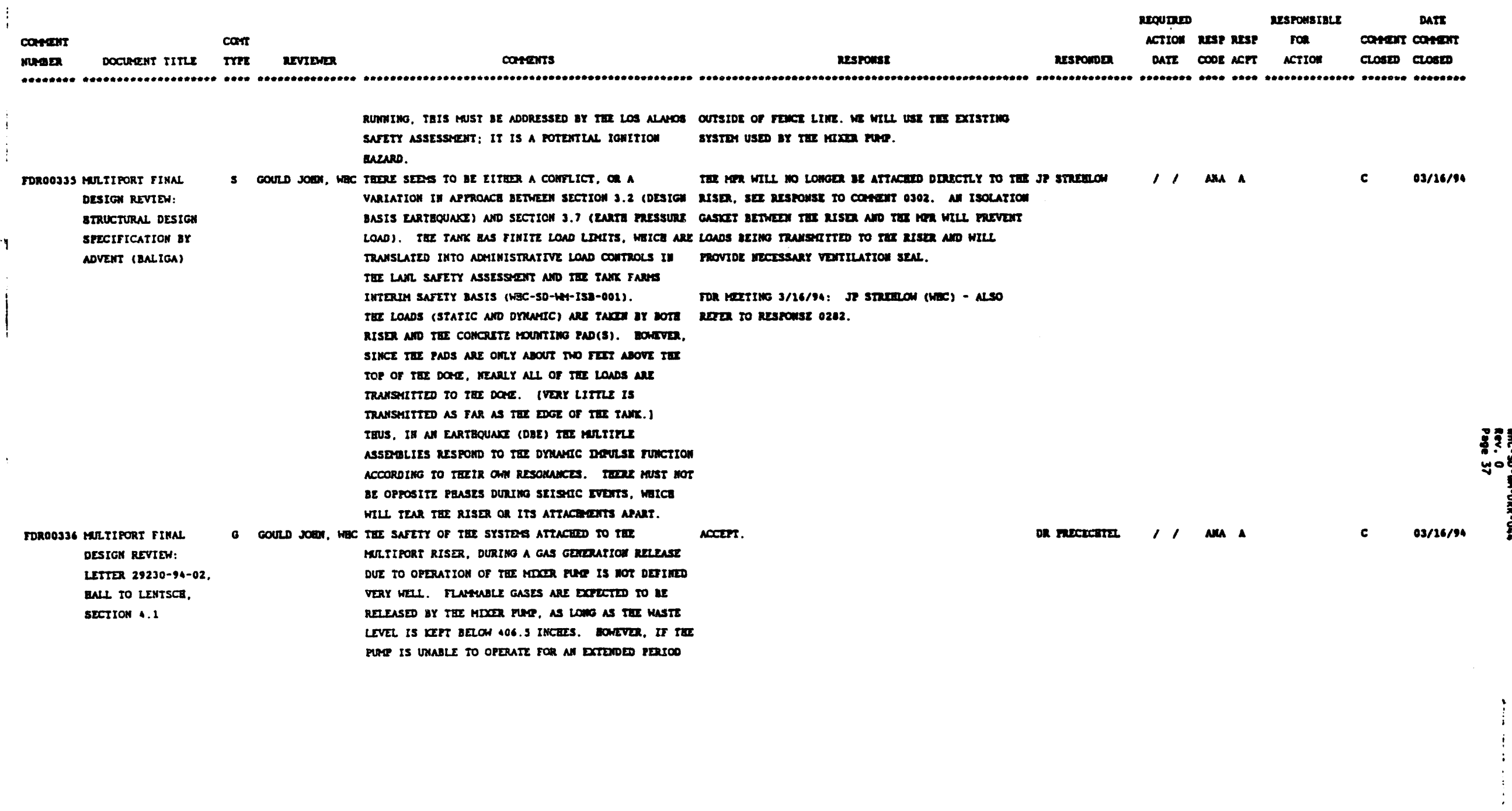


Pase No. 29

08/01/94

mattiport RISER

IIMAL DESTGM REVIEN

CONEETT DATALASE

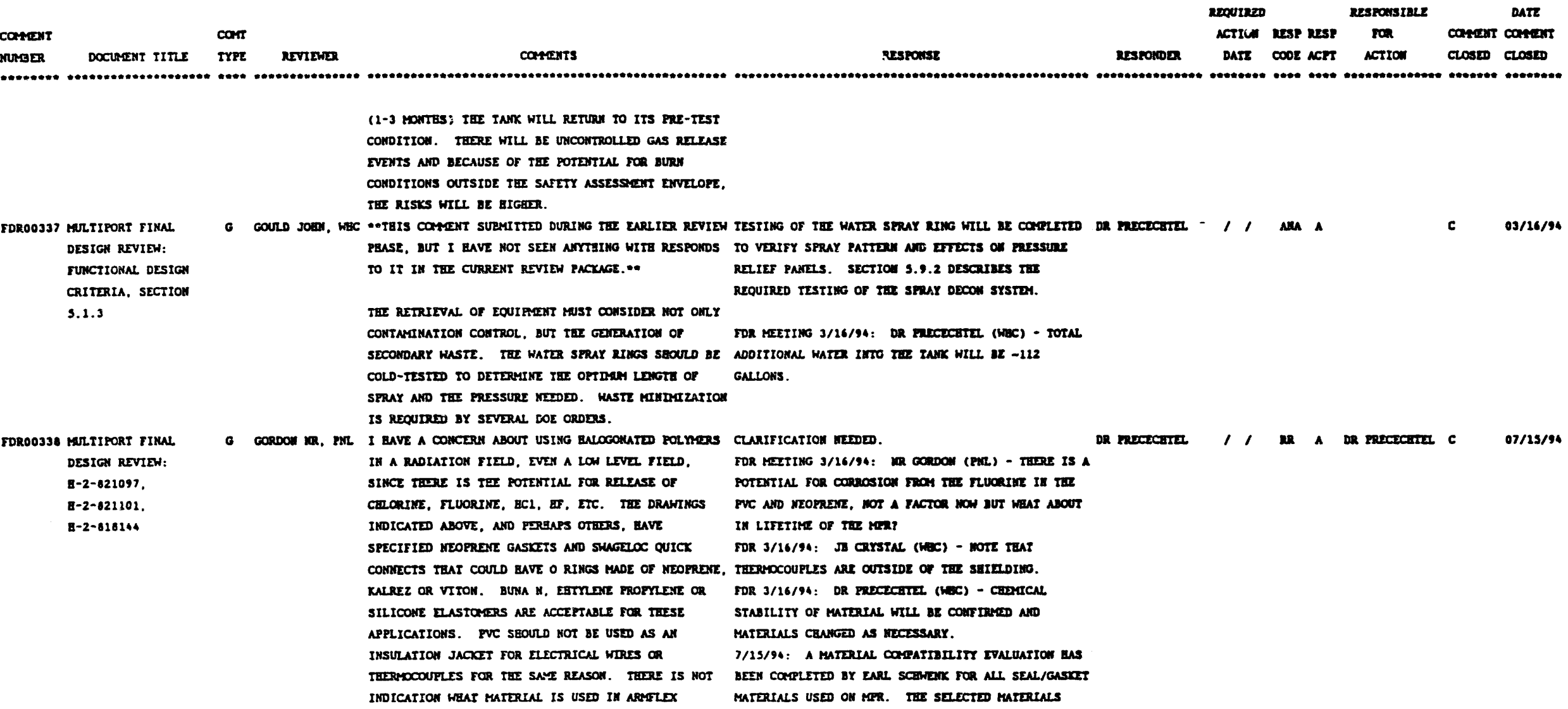


Pase No. 26

$08 / 01 / 96$

Martiport RISER

TIMNL DESICA REVIEW

coryext DataBnst

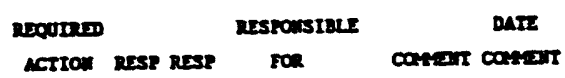

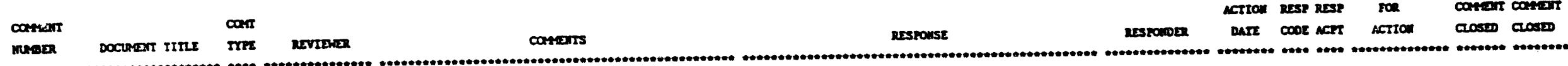

cascorss.

MIRE FONBD ACCEPTABLE.

FDR00339 MaLTIPORT FTKN

DESTG REvit:

B-2-121100, PAGE 2

Gussets no. 6

(1) TD200340 RartIPORI FINNL.

DESIGN REVIEN:

at-2-821100

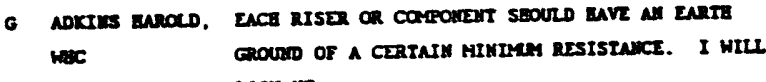
Loox ur.

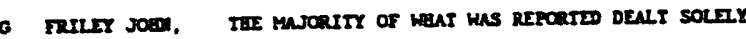
$m$

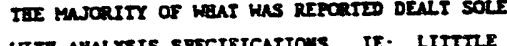

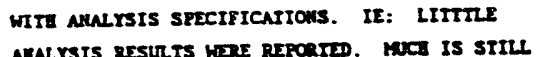
NRALYSIS EESURTS HER REPORTD. HCE IS STILL

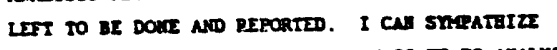

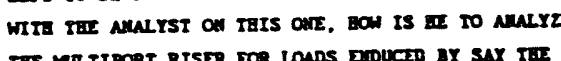

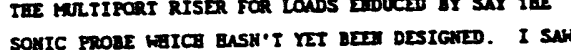

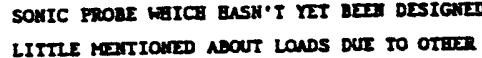

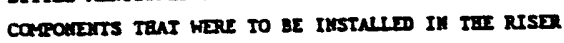
IN PNRTICURAR, RISER CORPOURTS WILL CAUSE

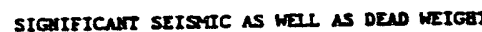

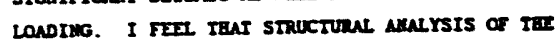

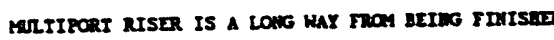

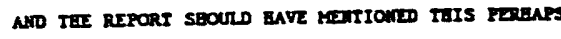
MORE OPEXI

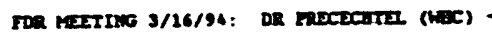

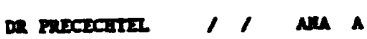

$03 / 16 / 9$

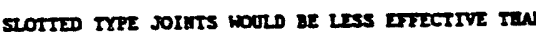

TII RIDCED JOIMTS. WE WIIL REVIEN TII ORTIDes of Artacterems.

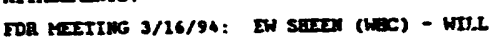
s carstas

1

m

a carst

c

$07 / 25 / 90$

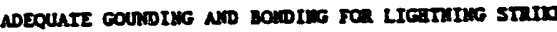

DerRmo Instnentiont

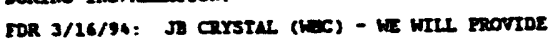

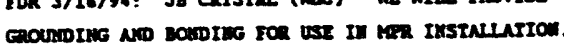

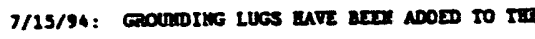

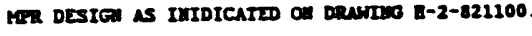

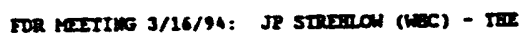

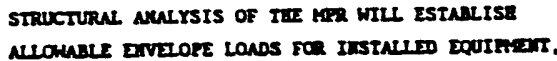

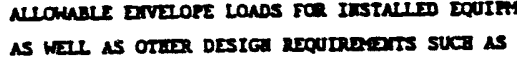

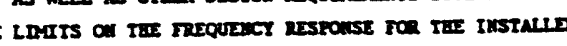

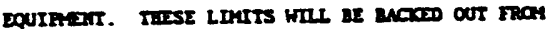

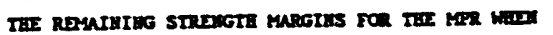

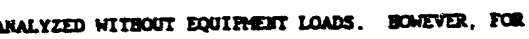

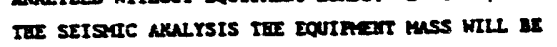

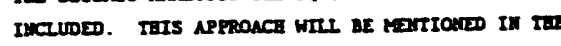
SDS FOR IEX HER. 
WHC-SD-WM-DRR-044

Rev. 0

Page 40

ATTACHMENT 3 
From: TWRS Safety Programs Engineering Support

Phone: $\quad 376-9507$ (L6-37)

Date: May 11, 1994

Subject: APPROVAL FOR FABRICATION OF MULTIPORT RISER ASSEMBLY

To: $\quad$ MPR DESIGN REVIEW COMMITTEE
H. E. Adkins
$\mathrm{N} 1-45$
R. E. Larson
$52-24$
J. R. Biggs
T4-01
R. S. Marlow
H5-56
L. E. Efferding
R. E. Mendoza
R1-51
J. R. Friley
$\mathrm{HO}-33$
M. L. McElroy
S1-57
N. R. Gordon
K2-44
L. M. McWethy
L6-37
J. I. Gould
H6-30
C. C. Scaief
L7-06
J. J. Klos
L. S. Krogsrud
T4-07
E. M. Sheen
L7-03
cc: R. Baliga
R3-08
M. A. Clayton
(ADVENT ENGR)
J. R. White
(LANL)
W. 0. Miller
D. R. Precechtel $\lfloor 6-37$
J. B. Crystal N1-42
J. R. Frederickson $\mathrm{Nl}-42$
C. E. Hanson H5-09
J. P. Strehlow H5-56
$\mathrm{LMM} / \mathrm{F}$ i le/LB

The design of the multiport riser assembly (MPR) is complete. Per the agreements at the design review meeting, the design can be released for fabrication after the structural analyses are satisfactorily completed.

The results of the structural analyses are reviewed in Attachment One. These analyses demonstrate that the MPR (with necessary modifications) satisfies all structural requirements.

The design review checklist from the final design review was completed by a few of the review committee members. This checklist is shown in Attachment Two.

The design review committee members will be asked to sign the approval form (Attachment Three) after concurring with the structural analyses results (Attachment One) and the design review checklist (Attachment Two).

Please call Mike McWethy (376-9507) or leave a CC:mail message when your review is complete, you concur that the design can be released for fabrication, and you are ready to sign the approval form.

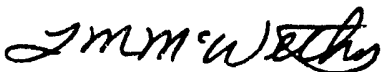

L. M. McWethy, Secretary

MPR 100\% Design Review Committee

APPROVED:

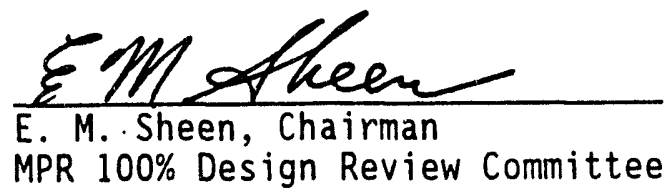




\section{ATTACHMENT ONE}

\section{MPR STRUCTURAL DESIGN SUMMARY}

Memo, J. P. Strehlow to E. M. Sheen, "Advent Structural Analysis of the Multiport Riser for Tank 241 SY-101," dated May 10, 1994. 
From: J. P. Strehlow

Phone: $376-5009$

Date: May 10, 1994

Subject: ADVENT STRUCTURAL ANALYSIS OF THE MULTIPORT RISER FOR TANK 241 SY-101

To: $\quad$ E. M. Sheen
cc: R. K. Brown
H5-68
A. W. Graves
L7-03
C. E. Hanson
H5- 09
D. R. Prechectel
L6-37

ADVENT Engineering has completed its structural analysis of the multiport riser that will be installed on tank 241 SY-101. Results from the analysis show that, to be fully acceptable for all load cases, several modifications are needed for the design presented in the $3 / 16 / 94$ final design review. These modifications have been reviewed and approved by the cognizant engineer, D.R. Precechtel, and have been incorporated into Revision $A$ of the design drawings.

The modifications to the multiport riser corrected over-stress problems that occurred in several plates and welds and an over-load problem with the concrete anchor bolts. All these structural problems were discovered in the Hydrogen burn load case evaluation. The design changes involved increasing plate thicknesses, making welds full penetration, and replacing the anchor bolts with a different type of anchor that offers a greater tensile load capacity. The following is a detailed list of these design changes.

- On drawing H-2-821095, Part 6, the base plate thickness was changed from 0.75 in. to 1.25 in.

- On drawing H-2-82095, Part 1, the support sub assembly thickness was changed from $1 / 2$ in. to $5 / 8$ in., a plate was added to the sub assembly, and the connection welds between the base plate, Part 6 , and the remainder of the support sub assembly were changed to full-penetration welds.

- On drawing H-2-821100, Part 23, the concrete anchor bolts were changed from HILTI HSLB M-20/30 to DRILLCO Maxi-Bolts MB-1250-30-23, ASTM A193, and the minimum edge distance for these anchor bolts added. Note 2 of this drawing has also been revised to address specific installation requirements such as torque and embedment depth. These anchors are not listed in SDC 4.2 , but they are fully compatible with DOE 6430.1A, General Design Requirements and they are used extensively throughout out the commercial nuclear industry. As such, it should be possible to purchase these anchors as safety class 1 items. 
WHC-SD-WM-DRR- 044

Rev. 0

E. M. Sheen

Page 2

May 10, 1994

- On drawing H-2-821101, sheets 4 and 5, Part 34, the thickness for the side plates of the multipart was changed from $1 / 2$ in. to $3 / 4$. and the welds that connect it to the bottom triangular plate, Part 35 , and to the top circular plate, part 37, were changed to fuli-penetration welds.

- On drawing $\mathrm{H}-2-82110$, sheet 6 , the welds between the upper flange on the support legs to the top circular plate, Part 37 , were changed to full-penetration welds.

ADVENT's structural analysis report for the multipart riser will be issued for WHC reviews on May 16. These reviews will include an independent review in accordance with engineering practices EP -4.1 and reviews by Safety and Quality Assurance

Gown Pthethow

John P. Strehlow Principal Engineer

TWRS Engineering Support Services

$\mathrm{scr}$ 


\section{ATTACHMENT TWO}

\section{DESIGN REVIEW CHECKLIST \\ FOR MULTIPORT RISER \\ FINAL DESIGN REVIEW}

Document $(s)$ Reviewed: MULTIPORT RISER FINAL DESIGN REVIEW J.R. Frederickson Corres. No. 9451465

\begin{tabular}{|c|c|c|c|c|c|}
\hline \multirow{2}{*}{$\begin{array}{c}\text { Project } \\
\text { Item }\end{array}$} & \multirow{2}{*}{$\begin{array}{l}\text { System/Component } \\
\text { Review Consideration }\end{array}$} & \multicolumn{3}{|c|}{ Design Phase } & \multirow{2}{*}{$\begin{array}{r}\text { Cognizant } \\
\text { Engineer } \\
\text { Remarks } \\
\end{array}$} \\
\hline & & Yes & No & NA & \\
\hline 1 & $\begin{array}{l}\text { Have assumptions necessary to } \\
\text { perform the design task been } \\
\text { adequately described and are } \\
\text { they reasonable? Where } \\
\text { necessary, have assumptions } \\
\text { been identified for reverifi- } \\
\text { cation when the design task has } \\
\text { been completed? }\end{array}$ & $x$ & & & \\
\hline 2 & $\begin{array}{l}\text { Were sources of information } \\
\text { identified? }\end{array}$ & $x$ & & & \\
\hline 3 & $\begin{array}{l}\text { Does the design meet the } \\
\text { established requirements or } \\
\text { design criteria? }\end{array}$ & $x$ & & & $\begin{array}{l}\text { Structural } \\
\text { analys is results } \\
\text { needed }\end{array}$ \\
\hline 4 & $\begin{array}{l}\text { Does the design meet } \\
\text { established requirements for } \\
\text { associated system physical and } \\
\text { functional interfaces? }\end{array}$ & $x$ & & & \\
\hline 5 & $\begin{array}{l}\text { Have the interface requirements } \\
\text { with site construction drawings } \\
\text { been clearly specified and are } \\
\text { they achievable? }\end{array}$ & $x$ & & & \\
\hline 6 & $\begin{array}{l}\text { Are there any interface } \\
\text { problems? }\end{array}$ & $x$ & & & \\
\hline
\end{tabular}


FINAL DESIGN REVIEW

\begin{tabular}{|c|c|c|c|c|c|}
\hline \multirow{2}{*}{$\begin{array}{r}\text { Project } \\
\text { Item }\end{array}$} & \multirow{2}{*}{$\begin{array}{l}\text { System/Component } \\
\text { Review Consideration }\end{array}$} & \multicolumn{3}{|c|}{ Design Phase } & \multirow{2}{*}{$\begin{array}{r}\text { Cognizant } \\
\text { Engineer } \\
\text { Remarks } \\
\end{array}$} \\
\hline & & Yes & No & NA & \\
\hline 7 & $\begin{array}{l}\text { Has appropriate consideration } \\
\text { been given to use of } \\
\text { standardized parts, materials, } \\
\text { and processes, and have } \\
\text { engineering standards and } \\
\text { criteria been specified } \\
\text { properly in the design? }\end{array}$ & $x$ & \multirow{9}{*}{\multicolumn{2}{|c|}{$x$}} & \\
\hline 8 & $\begin{array}{l}\text { Does the design represent the } \\
\text { simplest design consistent } \\
\text { with functional requirements } \\
\text { and expected service } \\
\text { conditions? }\end{array}$ & $x$ & & & . \\
\hline 9 & $\begin{array}{l}\text { Are the specified materials } \\
\text { compatible with each other and } \\
\text { the environmental conditions } \\
\text { to which the material will be } \\
\text { exposed? }\end{array}$ & $x$ & & & \\
\hline 10 & $\begin{array}{l}\text { Have available data on } \\
\text { similar designs been used? }\end{array}$ & \multirow{6}{*}{$x$} & & & \\
\hline \multirow[t]{5}{*}{11} & $\begin{array}{l}\text { Does the design meet } \\
\text { functional requirements? }\end{array}$ & & & & \\
\hline & $\begin{array}{l}\text { a. Stresses are within } \\
\text { design limits? }\end{array}$ & & & & $\begin{array}{l}\text { Structural } \\
\text { analysis results } \\
\text { needed }\end{array}$ \\
\hline & b. Derating is used? & & & & \\
\hline & $\begin{array}{l}\text { c. Steady-state and } \\
\text { transient conditions? }\end{array}$ & & & & $\begin{array}{l}\text { Structural } \\
\text { analysis results }\end{array}$ \\
\hline & $\begin{array}{l}\text { d. Have actual and "Worst } \\
\text { case" condition } \\
\text { stresses been con- } \\
\text { sidered rather than } \\
\text { nominal average } \\
\text { stresses? }\end{array}$ & & & & $\begin{array}{l}\text { Structural } \\
\text { analysis results } \\
\text { needed }\end{array}$ \\
\hline
\end{tabular}




\section{DESIGN REVIEW CHECKLIST \\ FOR MULTIPORT RISER \\ FINAL DESIGN REVIEW}

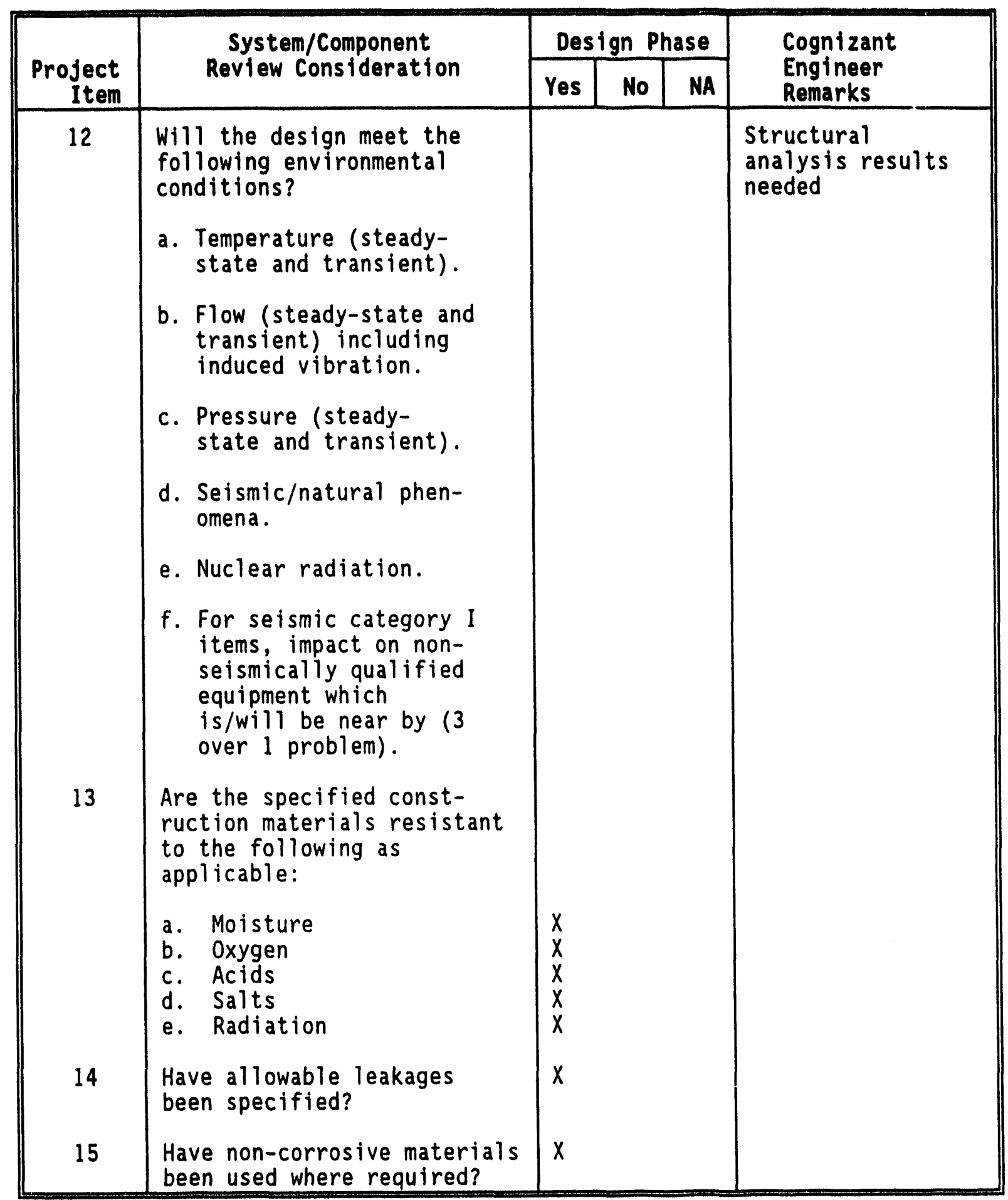




\begin{tabular}{|c|c|c|c|c|c|}
\hline \multirow{2}{*}{$\begin{array}{r}\text { Project } \\
\text { Item }\end{array}$} & \multirow{2}{*}{$\begin{array}{l}\text { System/Component } \\
\text { Review Consideration }\end{array}$} & \multicolumn{3}{|c|}{ Design Phase } & \multirow{2}{*}{$\begin{array}{r}\text { Cognizant } \\
\text { Engineer } \\
\text { Remarks } \\
\end{array}$} \\
\hline & & Yes & No & NA & \\
\hline 16 & $\begin{array}{l}\text { Does the design avoid any } \\
\text { materials unproven for use in } \\
\text { the anticipated environment? }\end{array}$ & $x$ & & & \\
\hline 17 & $\begin{array}{l}\text { Has the design appropriately } \\
\text { considered maintenance, } \\
\text { operation and reliability, } \\
\text { including maintenance } \\
\text { procedures and techniques, } \\
\text { unique maintenance } \\
\text { requirements and frequencies? }\end{array}$ & $x$ & & & \\
\hline 18 & $\begin{array}{l}\text { Has adequate accessibility } \\
\text { been provided for in service } \\
\text { inspection? }\end{array}$ & $x$ & & & \\
\hline 19 & $\begin{array}{l}\text { Does the design meet all } \\
\text { established safety } \\
\text { requirements? }\end{array}$ & $x$ & & & \\
\hline 20 & $\begin{array}{l}\text { Has an acceptable level of } \\
\text { radiation exposure been } \\
\text { defined? }\end{array}$ & $x$ & & & \\
\hline 21 & $\begin{array}{l}\text { Have personnel radiation } \\
\text { protection requirements been } \\
\text { considered and identified? }\end{array}$ & $x$ & & & \\
\hline 22 & $\begin{array}{l}\text { Have necessary features been } \\
\text { provided to maintain personnel } \\
\text { radiation exposure as low as } \\
\text { reasonably achievable? }\end{array}$ & $x$ & & & \\
\hline 23 & $\begin{array}{l}\text { Can the hardware be adeq- } \\
\text { uately disposed of after use } \\
\text { if it is radiologically or } \\
\text { chemically contaminated? }\end{array}$ & & & $x$ & \\
\hline
\end{tabular}


DESIGN REVIEW CHECKLIST

FOR MULTIPORT RISER

FINAL DESIGN REVIEW

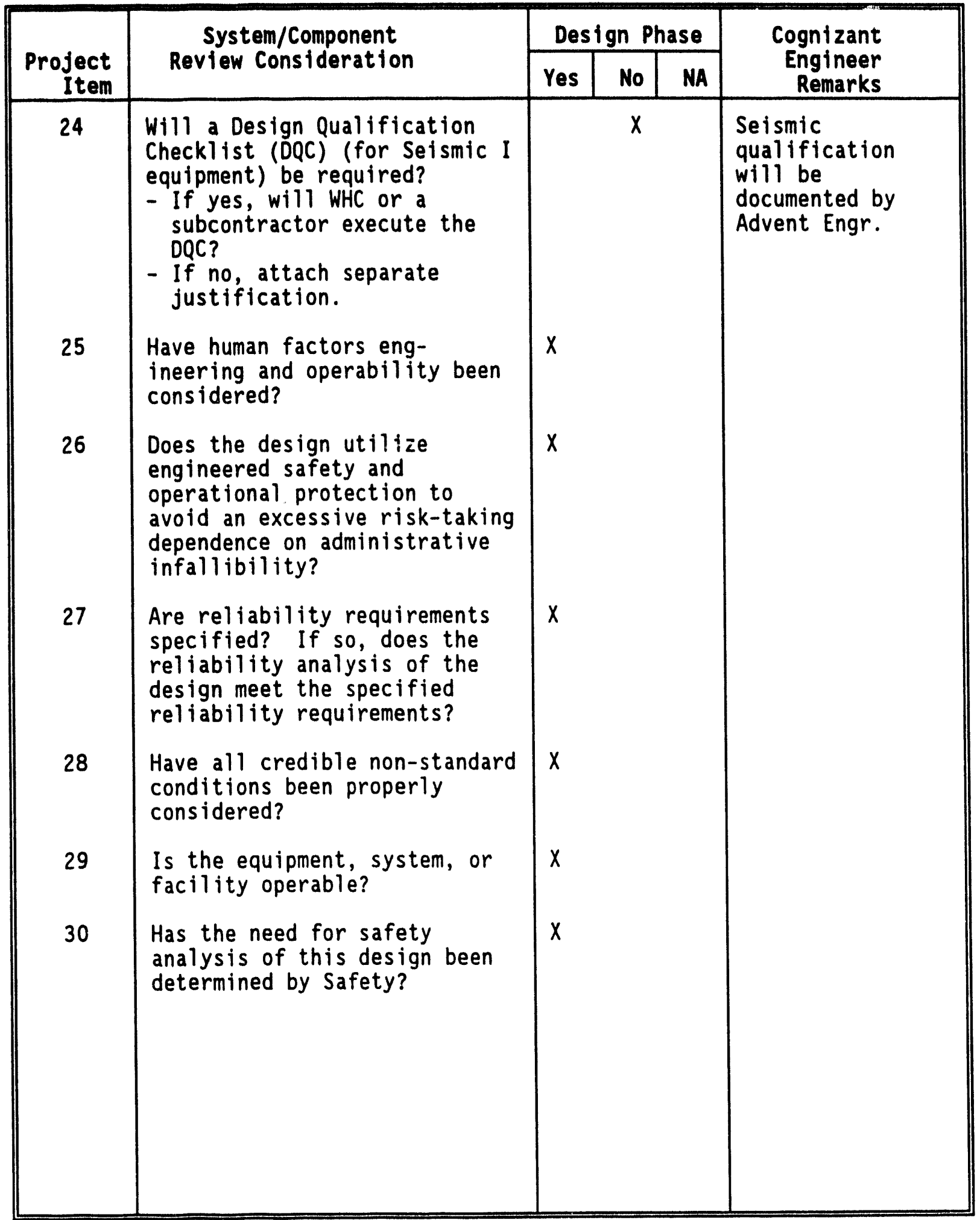




\section{DESIGN REVIEW CHECKLIST \\ FOR MULTIPORT RISER \\ FINAL DESIGN REVIEW}

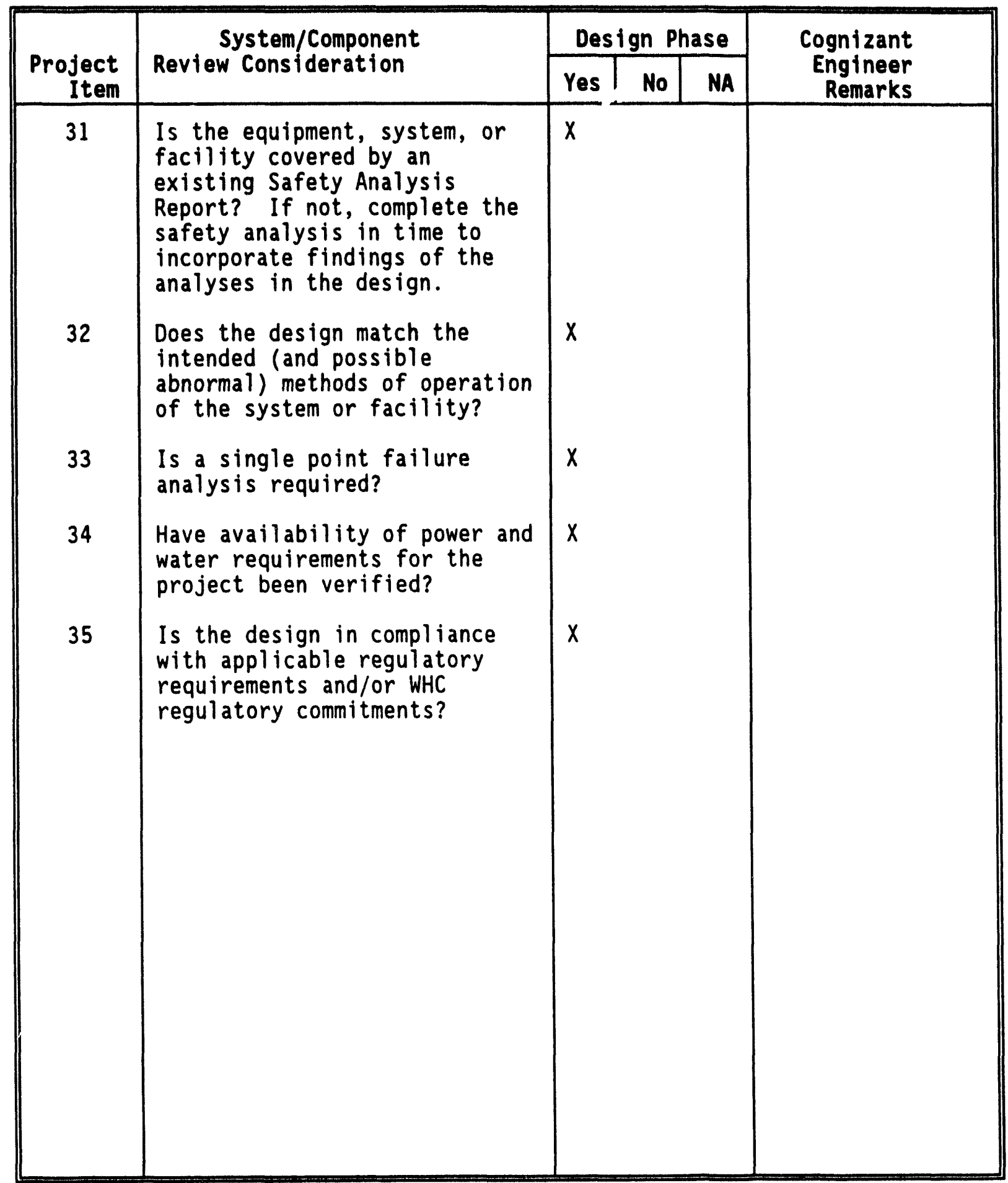


ATTACHMENT THREE

MULTIPORT RISER

DESIGN REVIEW COMMITTEE

APPROVAL FOR FABRICATION

\begin{tabular}{|l|l|l||}
\hline \multicolumn{1}{|c|}{ NAME } & \multicolumn{1}{|c|}{ FUNCTION } & SIGNATURE \\
\hline E. M. Sheen & Design Review Chairman & \\
\hline L. M. McWethy & Design Review Secretary & \\
\hline M. L. McElroy & Quality & \\
\hline L. S. Krogsrud & Safety & \\
\hline R. S. Marlow & Structural/Seismic & \\
\hline N. R. Gordon & Materials & \\
\hline J. J. Klos & Operations and Installation & \\
\hline L. E. Efferding & Thermal Hydraulic & \\
\hline J. I. Gould & Environmental & \\
\hline C. C. Scaief & I\&C Design & \\
\hline J. R. Friley & Acoustics and Sonic Transmission & \\
\hline R. E. Larson & Electrical Design & \\
\hline H. E. Adkins & Equip. Operability and Fabricability & \\
\hline R. E. Mendoza & Equipment and Software Testing & \\
\hline J. R. Biggs & Equipment Installation & \\
\hline
\end{tabular}


WHC - SD - WN-DRR - 044

Rev. 0

Page 52

ATTACHMENT 4 
From: $\quad$ TWRS Safety Programs Engineering Support

Phone: $\quad 376-9507$ (L6-37)

Date: June 14, 1994

Subject: COMPLETION OF MULTIPORT FINAL DESIGN REVIEW

To: $\quad$ MPR DESIGN REVIEW COMMITTEE

$\begin{array}{llll}\text { H. E. Adkins } & \text { N1-45 } & \text { R. E. Larson } & \text { S2-24 } \\ \text { J. R. Biggs } & \text { T4-01 } & \text { R. S. Marlow } & \text { H5-56 } \\ \text { L. E. Efferding } & \text { H0-33 } & \text { R. E. Mendoza } & \text { R1-51 } \\ \text { J. R. Friley } & \text { K5-22 } & \text { M. L. McElroy } & \text { S1-57 } \\ \text { N. R. Gordon } & \text { K2-44 } & \text { L. M. McWethy } & \text { L. } \\ \text { J. I. Gould } & \text { H6-30 } & \text { C. C. Scaief } & \text { L7-06 } \\ \text { J. J. Klos } & \text { T4-07 } & \text { E. M. Sheen } & \text { L7-03 }\end{array}$

cc:

R3-08

(Advent Engr) (w/o atts)

L6-37 (w/o atts)

N1-42 (w/O atts)

R. E. Bauer

M. A. Clayton

J. B. Crystal

$\mathrm{N} 1-42$ ( $w / 0$ atts)

C. E. Hanson

H5-09

W. O. Miller

(LANL)

D. R. Precechtel L6-37

J. P. Strehlow H5-56

J. R. White

LMM/File/LB

(LANL)

Reference: Memo, L. M. McWethy to MPR Design Review Committee, "Approval for Fabrication of Multiport Riser Assembly, "dated May 11, 1994.

The final design review of the multiport riser assembly was intended to close with approval to fabricate and completion of the design review checklist (above reference). Project management has since decided to proceed with fabrication, accepting the risk of any late design changes. This action removes fabrication approval from the design review committee's responsibilities. The last remaining item from the design review process is completion of the design review checklist. In the draft checklist (above reference), the incomplete responses reflected the lack of the structural analyses document at the time of the final design review (March 16, 1994). This analysis document is now completed and available for review.

To complete the formal design review process, the structural analysis document (Attachment 1) should be reviewed and the design review checklist completed. Please review the attached document relevant to the comments generated in the final design review (Attachment 2). The intent is to close these comments, if possible, based on the structural analyses results. This revised comment database and the design review checklist will become the 
MPR Design Review Committee

basis for the design review completion report. Please complete your review and comments by June 30 . This will allow the design review completion report to be completed for use in the operational readiness review on July 22.

Please respond with your approval and any comments via CC:mail.

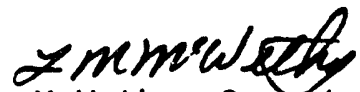

L. M. McWethy, Secretary

MPR 100\% Design Review Committee

Approved: \& Nil Cheen

E. M. Sheen, Chairman

MPR $100 \%$ Design Review Committee

$j k t$

Attachments 
WHC-SD-WM-DRR - 044

Rev. 0

Page 55

\section{ATTACHMENT 5}




\section{MULTIPORT RISER \\ DESIGN REVIEW COMMITTEE. APPROVAL}

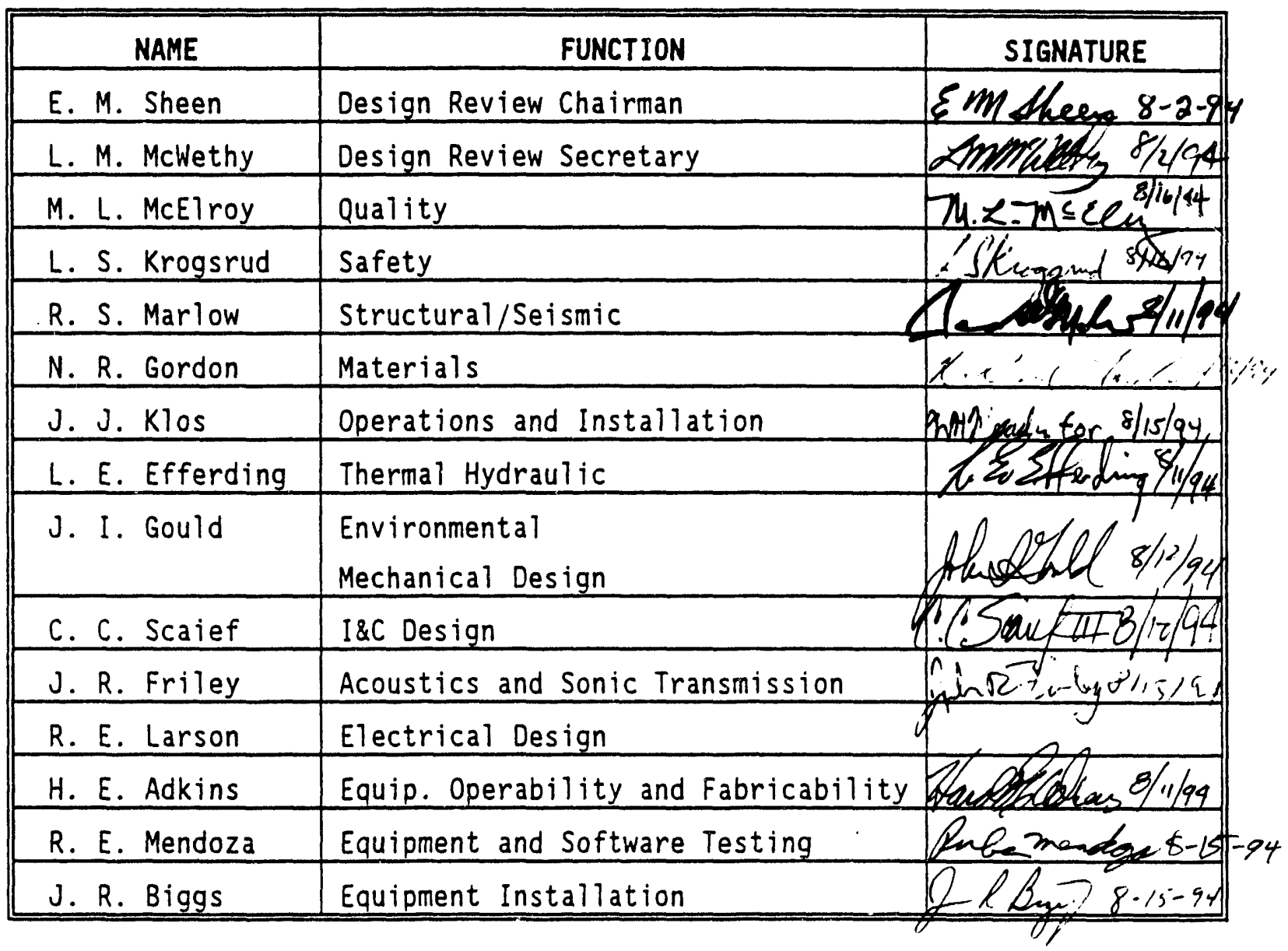




\section{DESIGN REVIEW CHECKLIST \\ FOR MULTIPORT RISER \\ FINAL DESIGN REVIEW}

Document(s) Reviewed:

MULTIPORT RISER FINAL DESIGN REVIEW

J.R. Frederickson

Corres. No. 9451465

\begin{tabular}{|c|c|c|c|c|c|}
\hline \multirow{2}{*}{$\begin{array}{c}\text { Project } \\
\text { Item }\end{array}$} & \multirow{2}{*}{$\begin{array}{c}\text { System/Component } \\
\text { Review Consideration }\end{array}$} & \multicolumn{3}{|c|}{ Design Phase } & \multirow{2}{*}{$\begin{array}{r}\text { Cognizant } \\
\text { Engineer } \\
\text { Remarks }\end{array}$} \\
\hline & & Yes & No & NA & \\
\hline 1 & $\begin{array}{l}\text { Have assumptions necessary to } \\
\text { perform the design task been } \\
\text { adequately described and are } \\
\text { they reasonable? Where } \\
\text { necessary, have assumptions } \\
\text { been identified for reverifi- } \\
\text { cation when the design task has } \\
\text { been completed? }\end{array}$ & $x$ & & & \\
\hline 2 & $\begin{array}{l}\text { Were sources of information } \\
\text { identified? }\end{array}$ & $x$ & & & \\
\hline 3 & $\begin{array}{l}\text { Does the design meet the } \\
\text { established requirements or } \\
\text { design criteria? }\end{array}$ & $x$ & & & \\
\hline 4 & $\begin{array}{l}\text { Does the design meet } \\
\text { established requirements for } \\
\text { associated system physical and } \\
\text { functional interfaces? }\end{array}$ & $x$ & & & \\
\hline 5 & $\begin{array}{l}\text { Have the interface requirements } \\
\text { with site construction drawings } \\
\text { been clearly specified and are } \\
\text { they achievable? }\end{array}$ & $x$ & & & \\
\hline 6 & $\begin{array}{l}\text { Are there any interface } \\
\text { problems? }\end{array}$ & & $x$ & & \\
\hline
\end{tabular}


FINAL DESIGN REVIEW

\begin{tabular}{|c|c|c|c|c|c|}
\hline \multirow{2}{*}{$\begin{array}{r}\text { Project } \\
\text { Item }\end{array}$} & \multirow{2}{*}{$\begin{array}{c}\text { System/Component } \\
\text { Review Consideration }\end{array}$} & \multicolumn{3}{|c|}{ Design Phase } & \multirow{2}{*}{$\begin{array}{r}\text { Cognizant } \\
\text { Engineer } \\
\text { Remarks }\end{array}$} \\
\hline & & Yes & No & NA & \\
\hline 7 & $\begin{array}{l}\text { Has appropriate consideration } \\
\text { been given to use of } \\
\text { standardized parts, materials, } \\
\text { and processes, and have } \\
\text { engineering standards and } \\
\text { criteria been specified } \\
\text { properly in the design? }\end{array}$ & $x$ & & & \\
\hline 8 & $\begin{array}{l}\text { Does the design represent the } \\
\text { simplest design consistent } \\
\text { with functional requirements } \\
\text { and expected service } \\
\text { conditions? }\end{array}$ & $x$ & & & \\
\hline 9 & $\begin{array}{l}\text { Are the specified materials } \\
\text { compatible with each other and } \\
\text { the environmental conditions } \\
\text { to which the material will be } \\
\text { exposed? }\end{array}$ & $x$ & & & \\
\hline 10 & $\begin{array}{l}\text { Have available data on } \\
\text { similar designs been used? }\end{array}$ & $x$ & & & \\
\hline 11 & $\begin{array}{l}\text { Does the design meet } \\
\text { functional requirements? }\end{array}$ & & & & \\
\hline & $\begin{array}{l}\text { a. Stresses are within } \\
\text { design limits? }\end{array}$ & $x$ & & & \\
\hline & b. Derating is used? & $x$ & & & \\
\hline & $\begin{array}{l}\text { c. Steady-state and } \\
\text { transient conditions? }\end{array}$ & $x$ & & & \\
\hline & $\begin{array}{l}\text { d. Have actual and "Worst } \\
\text { case" condition } \\
\text { stresses been con- } \\
\text { sidered rather than } \\
\text { nominal average } \\
\text { stresses? }\end{array}$ & $x$ & & & \\
\hline
\end{tabular}


DESIGN REVIEW CHECKLIST

FOR MULTIPORT RISER

FINAL DESIGN REVIEW

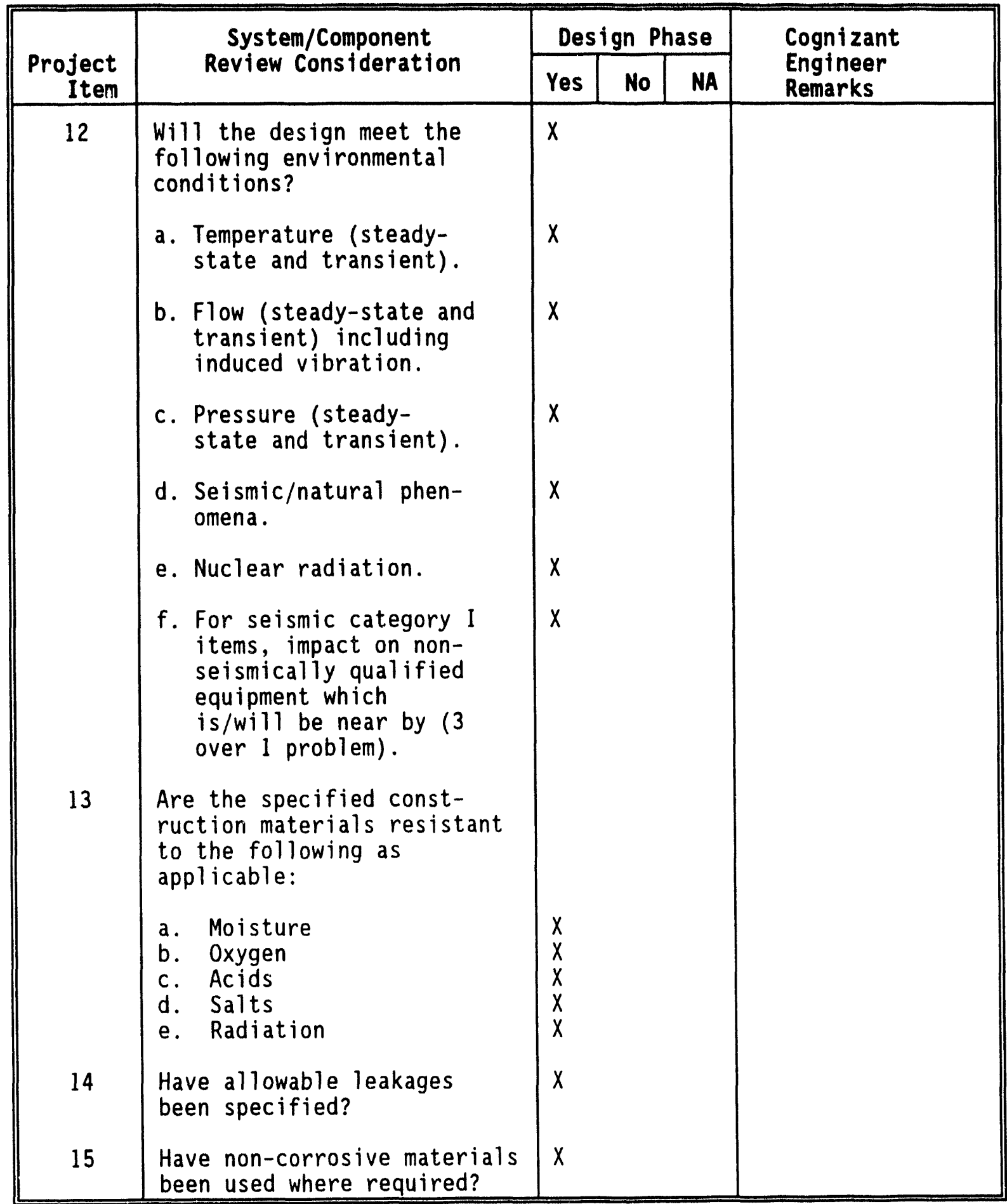


DESIGN REVIEW CHECKLIST

FOR MULTIPORT RISER

FINAL DESIGN REVIEW

\begin{tabular}{|c|c|c|c|c|c|}
\hline \multirow{2}{*}{$\begin{array}{r}\text { Project } \\
\text { Item } \\
\end{array}$} & \multirow{2}{*}{$\begin{array}{l}\text { System/Component } \\
\text { Review Consideration }\end{array}$} & \multicolumn{3}{|c|}{ Design Phase } & \multirow{2}{*}{$\begin{array}{r}\text { Cognizant } \\
\text { Engineer } \\
\text { Remarks } \\
\end{array}$} \\
\hline & & Yes & No & NA & \\
\hline 16 & $\begin{array}{l}\text { Does the design avoid any } \\
\text { materials unproven for use in } \\
\text { the anticipated environment? }\end{array}$ & $x$ & & & \\
\hline 17 & $\begin{array}{l}\text { Has the design appropriately } \\
\text { considered maintenance, } \\
\text { operation and reliability, } \\
\text { including maintenance } \\
\text { procedures and techniques, } \\
\text { unique maintenance } \\
\text { requirements and frequencies? }\end{array}$ & $x$ & & & \\
\hline 18 & $\begin{array}{l}\text { Has adequate accessibility } \\
\text { been provided for in service } \\
\text { inspection? }\end{array}$ & $x$ & & & \\
\hline 19 & $\begin{array}{l}\text { Does the design meet all } \\
\text { established safety } \\
\text { requirements? }\end{array}$ & $x$ & & & \\
\hline 20 & $\begin{array}{l}\text { Has an acceptable level of } \\
\text { radiation exposure been } \\
\text { defined? }\end{array}$ & $x$ & & & \\
\hline 21 & $\begin{array}{l}\text { Have personnel radiation } \\
\text { protection requirements been } \\
\text { considered and identified? }\end{array}$ & $x$ & & & \\
\hline 22 & $\begin{array}{l}\text { Have necessary features been } \\
\text { provided to maintain personnel } \\
\text { radiation exposure as low as } \\
\text { reasonably achievable? }\end{array}$ & $x$ & & & \\
\hline 23 & $\begin{array}{l}\text { Can the hardware be adeq- } \\
\text { uately disposed of after use } \\
\text { if it is radiologically or } \\
\text { chemically contaminated? }\end{array}$ & & & $x$ & \\
\hline
\end{tabular}




\section{DESIGN REVIEW CHECKLIST FOR MULTIPORT RISER FINAL DESIGN REVIEW}

\begin{tabular}{|c|c|c|c|c|c|}
\hline \multirow{2}{*}{$\begin{array}{r}\text { Project } \\
\text { Item }\end{array}$} & \multirow{2}{*}{$\begin{array}{l}\text { System/Component } \\
\text { Review Consideration }\end{array}$} & \multicolumn{3}{|c|}{ Design Phase } & \multirow{2}{*}{$\begin{array}{r}\text { Cognizant } \\
\text { Engineer } \\
\text { Remarks }\end{array}$} \\
\hline & & Yes & No & NA & \\
\hline 24 & $\begin{array}{l}\text { Will a Design Qualification } \\
\text { CheckIist (DQC) (for Seismic I } \\
\text { equipment) be required? } \\
\text { - If yes, will WHC or a } \\
\text { subcontractor execute the } \\
\text { DQC? } \\
\text { - If no, attach separate } \\
\text { justification. }\end{array}$ & & $x$ & & $\begin{array}{l}\text { Seismic } \\
\text { qualification was } \\
\text { documented by } \\
\text { Advent Engr. }\end{array}$ \\
\hline 25 & $\begin{array}{l}\text { Have human factors eng- } \\
\text { ineering and operability been } \\
\text { considered? }\end{array}$ & $x$ & & & \\
\hline 26 & $\begin{array}{l}\text { Does the design utilize } \\
\text { engineered safety and } \\
\text { operational protection to } \\
\text { avoid an excessive risk-taking } \\
\text { dependence on administrative } \\
\text { infallibility? }\end{array}$ & $x$ & & & \\
\hline 27 & $\begin{array}{l}\text { Are reliability requirements } \\
\text { specified? If so, does the } \\
\text { reliability analysis of the } \\
\text { design meet the specified } \\
\text { reliability requirements? }\end{array}$ & $x$ & & & \\
\hline 28 & $\begin{array}{l}\text { Have all credible non-standard } \\
\text { conditions been properly } \\
\text { considered? }\end{array}$ & $x$ & & & \\
\hline 29 & $\begin{array}{l}\text { Is the equipment, system, or } \\
\text { facility operable? }\end{array}$ & $x$ & & & \\
\hline 30 & $\begin{array}{l}\text { Has the need for safety } \\
\text { analysis of this design been } \\
\text { determined by Safety? }\end{array}$ & $x$ & & & \\
\hline
\end{tabular}




\section{DESIGN REVIEW CHECKLIST \\ FOR MULTIPORT RISER \\ FINAL DESIGN REVIEW}

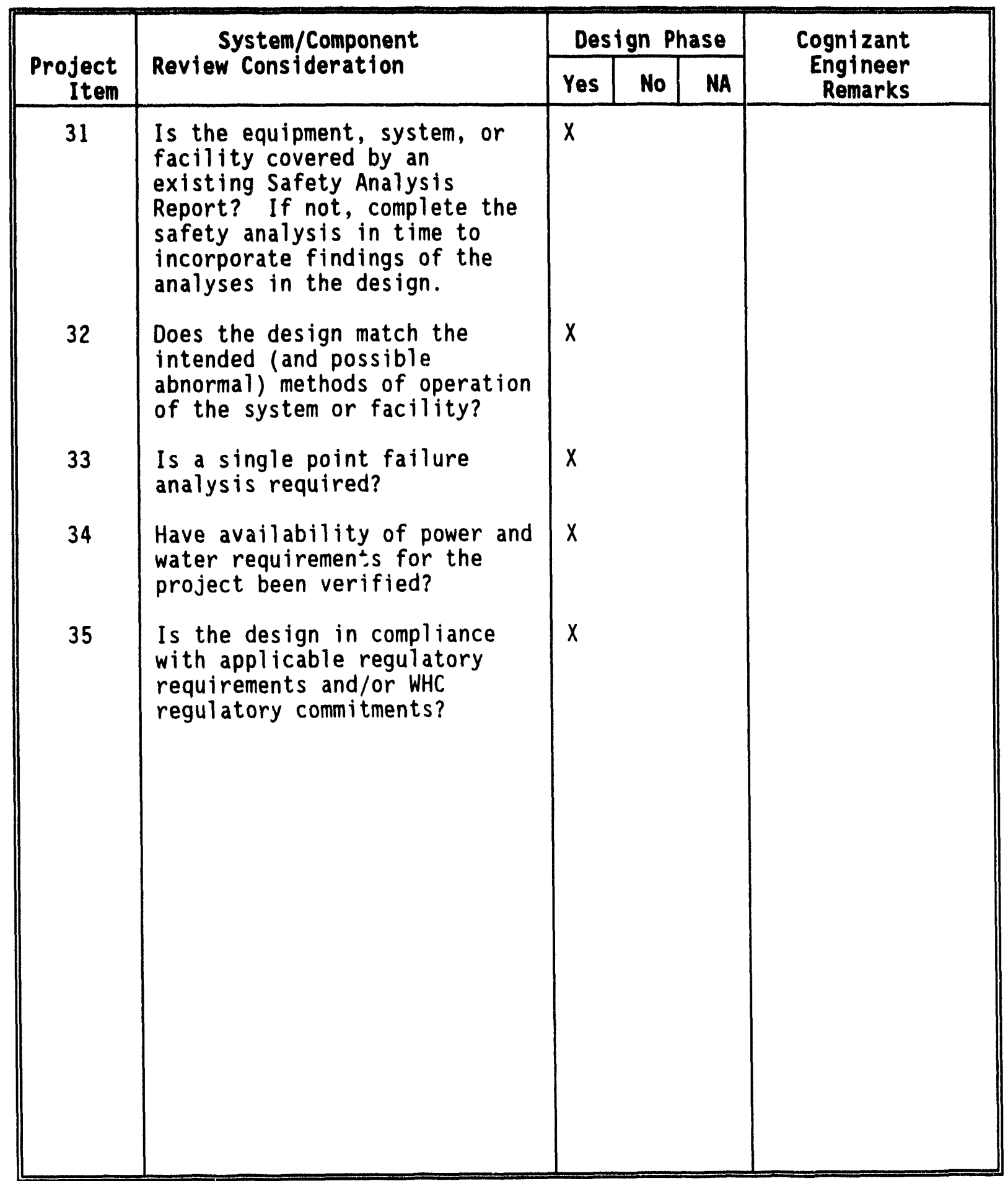




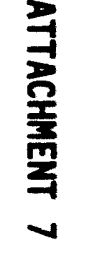




\section{MULTIPORT RISER \\ FINAL DESIGN REVIEW}

Wednesday, March 16, 1994

AGENDA

\section{8:00 a.m.}

\section{WeTcome - Introduction of Committee Members and Attenders \\ Opening Remarks}

Design Review Checklist Discussion

$8: 45 \mathrm{a} . \mathrm{m}$.

Safety Assessment Presentation

$9: 45$ a.m.

15 Minute Break

10:00 a.m.

Structural Analysis Presentation

11:00 a.m.

60\% Design Review Comment Database

Review - Resolve Open Items

$11: \frac{45 \mathrm{a} . \mathrm{m}}{30}$.

30 Minute Lunch Break - lunches provided in conference rooms.

$12: 20$ p.m.

Comment Database Review

Question and answer session with designers, engineers, and reviewers. Review comments and responses, discussion, and determine which comments are open and/or unresolved.

Multiport items

Mike McWethy - Secretary

2:00 D.m.

15 Minute Break

Continue Database Review

Mike McWethy - Secretary

Design Review Checklist Completion and Report Discussion

Wind up by $4: 00$ p.m.

\author{
Ed Sheen - Chairman \\ Jim Frederickson - Project \\ Manager \\ Ed Sheen - Chairman \\ Bob White - LANL
}

John Strehlow - WHC

Ravi Baliga - ADVENT

Ed Sheen - Chairman

Mike McWethy - Secretary 


\section{MULTIPORT RISER \\ DESIGN REVIEW COMITTEE}

For March 16, 1994 Review

at the 4862 building conference rooms

Beginning at 8:00 AM

Initial get together 3/1/94 at 8AM same place for briefing and packages.

\begin{tabular}{|c|c|c|}
\hline NAME & ORGANIZATION & FUNCTION \\
\hline EW Sheen & WHC - Central Engineering & $\begin{array}{l}\text { Design Review } \\
\text { Chairman }\end{array}$ \\
\hline LM McWethy & WHC - Remote Systems Development & $\begin{array}{l}\text { Design Review } \\
\text { Secretary }\end{array}$ \\
\hline ML McElroy & $\begin{array}{c}\text { WHC - Waste Tank Quality } \\
\text { Engineering }\end{array}$ & Quality \\
\hline LS Krogsrud & WHC - Waste Tank Safety & Safety \\
\hline RS Marlow & WHC - Strutural Margin Assesments & Structural/Seismic \\
\hline NR Gordon & PNL - Materials Sciences & Materials \\
\hline Ju Klos & WHC - Waste Tank Operations & $\begin{array}{l}\text { Operations and } \\
\text { Installation }\end{array}$ \\
\hline LE Efferding & WHC - Thermal Hydraulic Operations & Thermal Hydraul ic \\
\hline \multirow[t]{2}{*}{ JI Gould } & $\begin{array}{l}\text { WHC - Environmental Review and } \\
\text { Integration }\end{array}$ & Environmental \\
\hline & WHC - Mechanical Equipment & Mechanical Design \\
\hline CC Scaief & WHC - Instrumentation and Control & I \& C Design \\
\hline JR Friley & $\begin{array}{l}\text { PNL - Automation and Measurement } \\
\text { Science }\end{array}$ & $\begin{array}{l}\text { Acoustics and Sonic } \\
\text { Transmission }\end{array}$ \\
\hline RE Larson & WHC - Power Systems & Electrical Design \\
\hline HE Adkins & $\begin{array}{c}\text { WHC - Test Engineering and } \\
\text { Development }\end{array}$ & $\begin{array}{l}\text { Equip. Operability } \\
\text { and Fabricability }\end{array}$ \\
\hline RE Mendoza & WHC - Test Engineering & $\begin{array}{l}\text { Equipment and } \\
\text { Software Testing }\end{array}$ \\
\hline JR Biggs & WHC - West Area Operations & $\begin{array}{l}\text { Equipment } \\
\text { Installation }\end{array}$ \\
\hline
\end{tabular}

LANL declined to be on the Committee, but will attend. Lou Goldmann 
Rev. 0

Page 67

ATTACHMENT 8 


\section{Multiport Riser 100\% \\ Design Review (Design Packages) \\ Tuesday, March 1, 1994 at 8:00am \\ 4862 Bldg Conference RM}

Breaks As Needed

Welcome, introduction of committee members and attenders
Ed Sheen

Jim Frederickson

ICarl Hanson

$\begin{array}{ll}\text { Ed Sheen } & \text { Review Chairman } \\ \text { Ed Sheen } & \text { Review Chairman }\end{array}$

Project Manager
Review Chairman

Opening Remarks

Design Review Checklist Discussion

Comment Cata Base Review (Open Items)

Questions and answer session with designers, engineers, and reviewers. Review comments \& responses, discussion, and determine which comments are open and/or unresolved.

Multiport Items

Mike McWethy Review Secretary

\section{Presentation by Design Team}

- Multiport Mech. Design

- Multiport Elec. Design

- Overview of Dose Rate Calculations

- Structural Analysis

- Burn/Dome Structural Analysis

Closing Comments and Schedule for Design Review
Donald Precechtel

Jeremy Crystal

Randy Schwaz

John Strehlow

Bob White (LANL)

Ed Sheen 


\section{MULTIPORT RISER \\ DESIGN REVIEW COMMITTEE \\ 100\% DESIGN REVIEW}

For March 16, 1994 Review

at the 4862 Building Conference Rooms

Beginning at 8:00 a.m.

Initial get-together 3/1/94 at 8:00 a.m., same place for briefing and packages.

\begin{tabular}{|c|c|c|}
\hline NAME & ORGANIZATION & FUNCTION \\
\hline E. M. Sheen & WHC - Central Engineering & $\begin{array}{l}\text { Design Review } \\
\text { Chairman }\end{array}$ \\
\hline L. M. McWethy & WHC - Remote Systems Development & $\begin{array}{l}\text { Design Review } \\
\text { Secretary }\end{array}$ \\
\hline M. L. McElroy & $\begin{array}{c}\text { WHC - Waste Tank Quality } \\
\text { Engineering }\end{array}$ & Quality \\
\hline L. S. Krogsrud & WHC - Waste Tank Safety & Safety \\
\hline R. S. Marlow & WHC - Component Stress Analysis & Structural/Seismic \\
\hline N. R. Gordon & PNL - Materials Sciences & Materials \\
\hline J. J. Klos & WHC - Waste Tank Operations & $\begin{array}{l}\text { Operations and } \\
\text { Installation }\end{array}$ \\
\hline L. E. Efferding & $\begin{array}{l}\text { WHC - Thermal Hydraulic } \\
\text { Operations }\end{array}$ & Thermal Hydraulic \\
\hline J. I. Gould & $\begin{array}{c}\text { WHC - Environmental Review and } \\
\text { Integration }\end{array}$ & Environmental \\
\hline H. E. Adkins & $\begin{array}{c}\text { WHC - Test Engineering and } \\
\text { Development }\end{array}$ & $\begin{array}{l}\text { Mechanical Design, } \\
\text { Operability, \& } \\
\text { Fabricability }\end{array}$ \\
\hline C. C. Scaief & WHC - Instrumentation and Control & I\&C Design \\
\hline J. R. Friley & $\begin{array}{l}\text { PNL - Automation and Measurement } \\
\text { Science }\end{array}$ & $\begin{array}{l}\text { Acoustics and } \\
\text { Sonic Transmission }\end{array}$ \\
\hline R. E. Larson & WHC - Power Systems & Electrical Design \\
\hline R. E. Mendoza & WHC - Test Engineering & $\begin{array}{l}\text { Equipment and } \\
\text { Software Testing }\end{array}$ \\
\hline J. R. Biggs & WHC - West Area Operations & $\begin{array}{l}\text { Equipment } \\
\text { Installation }\end{array}$ \\
\hline
\end{tabular}


Here are the steps for the Multiport Final Design Review. For questions call Don Precechtel on (509) 376-3329 or Marci Clayton on (509) 376-8260.

\section{Responsibility Step Number Action}

\section{Design Reviewers}

Marci Clayton

Respondents

Marci Clayton
1 Please submit comments using the attached form. Using the form will ensure a more reliable entry of the comments into the Design Review Comment Database. The form is also available in electronic form via CC Mail. (Contact Marci Clayton)

Reference the comment by notebook, notebook section divider, page number, section number, paragraph number, or subject matter as appropriate.

Comments have to be received by Marci Clayton at N1-42, Fax (509) 373-0209, or by CC:Mail no later than COB March 14, 1994 for entry into the comment database.

2 Enter comments into the database and assign identifier numbers to the comments.

Distribute Comment Database to the respondents for comment answering and response.

3 Answer comments using the following response codes if possible. Respondent may need to provide text relating to proposed disposition of the comment.

$$
\begin{aligned}
A N A= & \text { Acknowledged comment, no action } \\
& \text { required } \\
I= & \text { Comment Incorporated } \\
\text { CRR }= & \text { Requires further clarification by } \\
& \text { Design Reviewer } \\
R R= & \text { Response Required } \\
R F R= & \text { Refer to Another Review } \\
N A= & \text { Not applicable }
\end{aligned}
$$

Return answers to Marci Clayton.

4 Answers are entered into the database and the database is presented at the Design Review for review and discussion on March 16, 1994. 
Review Chairman and Secretary

Marci Clayton

Review Secretary
5 Conduct review of the entire database at the design review meeting with the design reviewers for content arid acceptability.

Conduct discussion of unanswered comments and try to obtain closure of them, if possible. Remaining open comments are listed as open items in the database.

$6 \quad$ Updates the database as required and supplies it to the review secretary for inclusion into the design review minutes.

7 Publishes design review minutes after approval by the Design Review Committee. 
INSTRUCTIONS FOR DESIGN REVIEW COMMENT FORM

- Please type (cc:mail) or print form.

- $\quad T h e$ form is a table = TAB OR RETURN AT THE END OF EACH LINE.

- If you have any problems please contact Marci Clayton on 6-8260.

Design Review Comment Form

Reviewer

Name:

Location of item to which comment applies:
Page

Date:

Comment:

What type of comment is it? Check appropriate box.

$\mathbf{G}=$ General

$E=$ Editorial

$S=$ System Specific

[] $0=$ other, Please explain 


\section{COMMENT DATABASE STRUCTURE}

- All comments will be loaded into a database sequentially as they are recieved and the following fields will be printed:

- COMMENT NUMBER

$$
\text { FDR00001 = Final Design Review }
$$

- DOCUMENT TITLE

- COMMENT TYPE

$\mathbf{G}=$ General

$E=$ Editorial

S $=$ System Specific

$0=$ Other

- REVIEWER

- COMMENTS

- RESPONSE

- REQUIRED ACTION DATE

- RESPONSE CODE

$$
\begin{aligned}
& \text { ANA }=\text { Acknowledge, No Action Required } \\
& 1 \quad=\text { Incorporate } \\
& \text { CRR }=\text { Clarification Required from Reviewer } \\
& \text { RR }=\text { Response Required } \\
& \text { RFR }=\text { Refer to Another Review } \\
& \text { NA }=\text { Not Applicable }
\end{aligned}
$$

- RESPONSE ACCEPTED

- RESPONSIBLE FOR ACTION

- COMMENT ClOSED

- DATE ACTION ClOSED 
Pace No.

MILTIPOAT RISER

FIRAL DESION REVIEW

COARENT DATABASE

MEourges oute

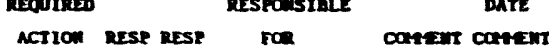
$\begin{array}{lll}\text { COFENT } & \text { COHT } \\ \text { MUREER DOCLNENT IITLE TYPE REVIEHER }\end{array}$ Contents RESPOUSE DATE COOE ACPT ACTIOH ClOSED CLOSED

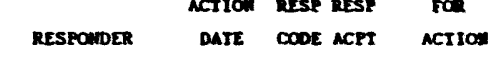

FDR00279 

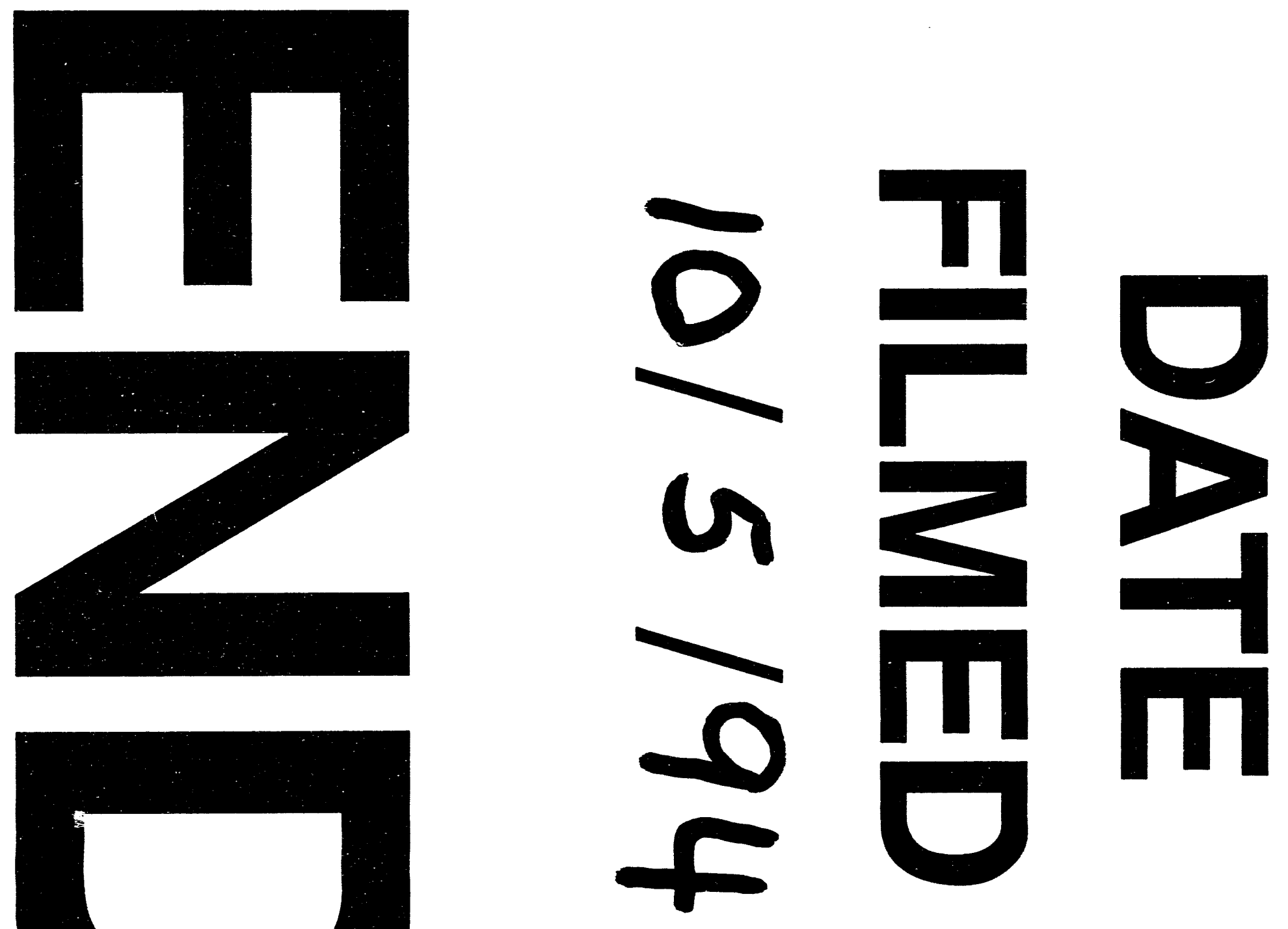
